\title{
A MORTAR EDGE ELEMENT METHOD WITH NEARLY OPTIMAL CONVERGENCE FOR THREE-DIMENSIONAL MAXWELL'S EQUATIONS
}

\author{
QIYA HU, SHI SHU, AND JUN ZOU
}

\begin{abstract}
In this paper, we are concerned with mortar edge element methods for solving three-dimensional Maxwell's equations. A new type of Lagrange multiplier space is introduced to impose the weak continuity of the tangential components of the edge element solutions across the interfaces between neighboring subdomains. The mortar edge element method is shown to have nearly optimal convergence under some natural regularity assumptions when nested triangulations are assumed on the interfaces. A generalized edge element interpolation is introduced which plays a crucial role in establishing the nearly optimal convergence. The theoretically predicted convergence is confirmed by numerical experiments.
\end{abstract}

\section{INTRODUCTION}

The main interest of this work is to explore some new mortar edge element methods for solving the following three-dimensional system:

$$
\operatorname{curl}(\alpha(x) \operatorname{curl} \mathbf{u})+\beta(x) \mathbf{u}=\mathbf{f}(x) \quad \text { in } \quad \Omega
$$

where $\Omega$ is an open polyhedral domain in $\mathbf{R}^{3}$, not necessarily convex, and the coefficients $\alpha(x)$ and $\beta(x)$ are two positive bounded functions in $\Omega$. The system (1.1) has to be solved repeatedly in numerical solutions of the Maxwell's equations [12, [15, [16, 19]. The equation (1.1) will be complemented with the following perfect conductor condition:

$$
\mathbf{u} \times \mathbf{n}=0 \quad \text { on } \quad \partial \Omega
$$

where $\mathbf{n}$ is the unit outward normal vector on $\partial \Omega$.

Both nodal and edge finite element methods have been used for solving the system (1.1)-(1.2); see, for example, [6], [13, [14], [15], 30]. It is well-known that the algebraic systems resulting from the discretization of the system (1.1)-(1.2) by the standard nodal and edge element methods differ greatly in nature. This

Received by the editor February 3, 2005 and, in revised form, March 28, 2007.

2000 Mathematics Subject Classification. Primary 65N30, 65N55.

Key words and phrases. Maxwell's equations, Nédélec finite elements, domain decomposition, nonmatching grids, generalized interpolation, error estimate.

The work of the first author was supported by the Natural Science Foundation of China G10371129, the Key Project of Natural Science Foundation of China G10531080, and the National Basic Research Program of China G2005CB321702.

The work of the second author was partially supported by the grant (2005CB321702).

The work of the third author was substantially supported by Hong Kong RGC grants (Project 404407 and Project 404606). 
difference leads to the construction of essentially different solvers based on different principles for the resulting discrete systems; see [11, 19, 20] and the references therein.

During the past decade, edge element methods have become increasingly more and more popular in solving the Maxwell equations (1.1)-(1.2). Many effective numerical solvers have been investigated for the discrete systems arising from the edge element discretizations; see [19], 11] for multigrid methods and [3], 18, [26, 27, 33, 34, 35] for domain decomposition methods. But all these multigrid and domain decomposition methods are built on a globally quasi-uniform grid on the whole domain $\Omega$. This certainly restricts the applications of these methods for the Maxwell system in nonhomogeneous media, where one may use independent grids in each medium region, which are nonmatching across the interface between any two neighboring medium regions, to achieve better performance of the numerical methods. The resulting methods of this type are often called mortar element methods. Clearly, the treatment of the nonmatching grids across interfaces is a central issue for a mortar element method to be accurate and effective. The DDMs based on nonmatching grids were widely studied for second order elliptic problems; see [8], 29, 22], 23], 28, 29] and [36]. Some DDMs with nonmatching grids were also investigated for two-dimensional Maxwell's equations in [1], 35, and for threedimensional Maxwell's equations like (1.1)-(1.2) in [7, 21. As it is well-known, the nonoverlapping domain decomposition theory for the nodal element systems, which has been well developed for second order elliptic problems in the past two decades (see the review article [37] and the references therein), do not work for the edge element systems in general, especially in three dimensions. Very little has been done with the convergence analysis of the mortar element methods for three-dimensional Maxwell's equations. The first important advance in this direction was made by Belgacem, Buffa and Maday in [7], and it is still the most significant work in the literature. But the results in [7] are a bit unsatisfactory as they used much higher regularity than expected to establish an energy-norm error estimate of their mortar element method for three-dimensional Maxwell's equations: in order to achieve a nearly first order accuracy $O\left(h(\log (h))^{1 / 2}\right)$ when the second family of Nedelec edge elements were used, it requires at least the regularity $\mathbf{u} \in H^{2}\left(\mathbf{c u r l} ; \Omega_{k}\right)$ in each subdomain $\Omega_{k}$; and no convergence is possible under the usual regularity assumption $\mathbf{u} \in H^{1}\left(\mathbf{c u r l} ; \Omega_{k}\right)$. If the first family of Nedelec edge elements was used, the convergence of the mortar edge element method of [7. would lose a further half order compared to the aforementioned convergence order of the second family. Another restriction of work [7] is that the ratio between the largest mesh size of all subdomains and the smallest one of all subdomains goes into the bound of the final error estimate.

To the best of our knowledge, there is still no convincing result in the literature on the construction and analysis of a mortar edge element method which possesses an optimal convergence in the energy-norm. In fact, it is still unknown whether it is possible to construct such an optimal mortar edge element method over general nonmatching finite element grids. The main difficulty for the approach used in the earlier effort [7] is that the $L^{2}$-norm of the jump of the tangential trace on a face cannot achieve any convergence order (see page 897 of [7]). Also, we think that the estimate of [7] cannot be improved unless an essentially different approach is taken. 
In this paper, we will make a further attempt to investigate the problem, and demonstrate that the construction of such optimal mortar edge element methods is indeed possible, at least for some nonmatching grids which are of certain nested structures on the interfaces between neighboring subdomains. We shall propose a mortar edge element method, and show that the method can achieve the nearly optimal error estimate of order $h^{\delta_{k}}(\log (d / h))^{1 / 2}$ in the energy-norm under the natural regularity conditions $\mathbf{u} \in H^{\delta_{k}}\left(\mathbf{c} u r l ; \Omega_{k}\right)$ in each subdomain $\Omega_{k}\left(\delta_{k}>1 / 2\right)$, when grids from two neighboring medium subdomains are assumed to be nested on their common interface. This assumption seems practical, since there are no efficient methods to calculate the integrations on the interface for general nonmatching grids (see 22 for such discussions on second order elliptic problems). Our mortar element method is based on the discretization of the Maxwell system (1.1)-(1.2) by the first family of edge elements (but it can be naturally extended to the easier case of the second family of edge elements). To overcome the difficulty that the standard saddle-point theory is not applicable for the error estimate of the proposed mortar edge element method, we shall develop a new approach, which relies heavily on an important generalized edge element interpolation.

The rest of this paper is arranged as follows. Some basic edge element spaces and definitions are described in Section 2, In Section 3, a mortar edge element method is proposed for the system (1.1)-(1.2) based on a new matching condition, and the unique solvability of the discrete problem is proved. Section 4 introduces and studies a generalized edge element interpolation operator. The main result of this paper about the optimal error estimates is analyzed in Section 5. Numerical experiments are presented in Section 6 to confirm the nearly optimal convergence of the mortar edge element method predicted by the convergence theory.

\section{Domain DECOMPOSITIONS AND DisCRETIZATIONS}

This section is devoted to the introduction of a nonoverlapping domain decomposition, a weak variational form and some basic edge element spaces.

Domain decomposition. We decompose the physical domain $\Omega$ into $N$ nonoverlapping tetrahedral subdomains $\left\{\Omega_{k}\right\}_{k=1}^{N}$, with each $\Omega_{k}$ of size $d$ (see [37]). The faces and vertices of the subdomains are always denoted by $\mathrm{F}$ and $\mathrm{V}$, while the common (open) face of the subdomains $\Omega_{i}$ and $\Omega_{j}$ are denoted by $\Gamma_{i j}$, and the union of all such common faces by $\Gamma$, i.e., $\Gamma=\bigcup \bar{\Gamma}_{i j}$. $\Gamma$ will be called the interface. By $\Gamma_{k}$ we denote the intersection of $\Gamma$ with the boundary of the subdomain $\Omega_{k}$. So we have $\Gamma_{k}=\partial \Omega_{k}$ if $\Omega_{k}$ is a subdomain lying strictly inside $\Omega$.

Finite element triangulation. Further, we divide each subdomain $\Omega_{k}$ into smaller tetrahedral elements of size $h_{k}$. The resulting triangulation of the domain $\Omega_{k}$ is denoted by $\mathcal{T}_{h_{k}}$, which is assumed to be quasi-uniform (cf. [37]), while the set of edges and the set of nodes in $\mathcal{T}_{h_{k}}$ are denoted by $\mathcal{E}_{h_{k}}$ and $\mathcal{N}_{h_{k}}$, respectively. The triangulations in the subdomains generally do not match on the interfaces between subdomains. Hence, each interface $\Gamma_{i j}$ inherits two triangulations $\mathcal{T}_{i j}$ and $\mathcal{T}_{j i}$, which are naturally induced from $\mathcal{T}_{h_{i}}$ and $\mathcal{T}_{h_{j}}$, respectively. We shall use $h$ to denote the fine mesh size over $\Omega$, i.e. $h=\min _{1 \leq k \leq N} h_{k}$.

Weak formulation. The primary goal of this paper is to construct an efficient mortar edge element method for solving the equation (1.1). For this, we first introduce its weak form and then the edge element discretization of the weak form. Let $H(\mathbf{c u r l} ; \Omega)$ be the Sobolev space consisting of all square integrable functions 
whose curl's are also square integrable in $\Omega$, and $H_{0}(\mathbf{c u r l} ; \Omega)$ be a subspace of $H(\operatorname{curl} ; \Omega)$ with all functions whose tangential components vanish on $\partial \Omega$, i.e., $\mathbf{v} \times$ $\mathbf{n}=0$ on $\partial \Omega$ for all $\mathbf{v} \in H_{0}(\mathbf{c u r l} ; \Omega)$. Then by integration by parts, one derives immediately the variational problem associated with the system (1.1)-(1.2): Find $\mathbf{u} \in H_{0}(\operatorname{curl} ; \Omega)$ such that

$$
A(\mathbf{u}, \mathbf{v})=(\mathbf{f}, \mathbf{v}), \quad \forall \mathbf{v} \in H_{0}(\mathbf{c u r l} ; \Omega)
$$

where $A(\cdot, \cdot)$ is a bilinear form given by

$$
A(\mathbf{u}, \mathbf{v})=(\alpha \operatorname{curl} \mathbf{u}, \operatorname{curl} \mathbf{v})+(\beta \mathbf{u}, \mathbf{v}), \quad \forall \mathbf{u}, \mathbf{v} \in H(\operatorname{curl} ; \Omega) .
$$

Here and in what follows, $(\cdot, \cdot)$ denotes the scalar product in $L^{2}(\Omega)$ or $\left(L^{2}(\Omega)\right)^{3}$.

Edge element discretization. For each subdomain $\Omega_{k}$, we introduce the Nédélec edge element space, of the lowest order, which is a subspace of piecewise linear polynomials defined on $\mathcal{T}_{h_{k}}$ (cf. [31]):

$$
V_{h_{k}}\left(\Omega_{k}\right)=\left\{\mathbf{v} \in H_{0}(\operatorname{curl} ; \Omega) ;\left.\mathbf{v}\right|_{K} \in R(K), \forall K \in \mathcal{T}_{h_{k}}\right\}
$$

where $R(K)$ is a subset of all linear polynomials on the element $K$ of the form:

$$
R(K)=\left\{\mathbf{a}+\mathbf{b} \times \mathbf{x} ; \mathbf{a}, \mathbf{b} \in \mathbf{R}^{3}, \mathbf{x} \in K\right\} .
$$

It is known from [31] that the tangential components of any function $\mathbf{v}$ in $V_{h_{k}}\left(\Omega_{k}\right)$ are continuous on all edges of every element in the triangulation $\mathcal{T}_{h_{k}}$, and $\mathbf{v}$ is uniquely determined by its moments on the edges of $\mathcal{T}_{h_{k}}$ :

$$
\left\{\lambda_{e}(\mathbf{v})=\int_{e} \mathbf{v} \cdot \mathbf{t}_{e} d s ; e \in \mathcal{E}_{h_{k}}\right\}
$$

where $\mathbf{t}_{e}$ denotes the unit vector on the edge $e$.

Let $\left\{L_{e} ; e \in \mathcal{E}_{h_{k}}\right\}$ be the edge element basis functions of $V_{h_{k}}\left(\Omega_{k}\right)$ satisfying

$$
\lambda_{e^{\prime}}\left(L_{e}\right)= \begin{cases}1, & \text { if } e^{\prime}=e, \\ 0, & \text { otherwise }\end{cases}
$$

One can verify that the edge element basis function $L_{e}$ associated with the edge $e$ has the representation

$$
L_{e}=c^{e}\left(\lambda_{1}^{e} \nabla \lambda_{2}^{e}-\lambda_{2}^{e} \nabla \lambda_{1}^{e}\right),
$$

where $\lambda_{1}^{e}$ and $\lambda_{2}^{e}$ are two barycentric basis functions at two endpoints of $e$, and $c^{e}$ is a constant such that $\lambda_{e}\left(L_{e}\right)=1$. Also, each function $\mathbf{v}$ of $V_{h_{k}}\left(\Omega_{k}\right)$ can be expressed as

$$
\mathbf{v}(\mathbf{x})=\sum_{e \in \mathcal{E}_{h_{k}}} \lambda_{e}(\mathbf{v}) L_{e}(\mathbf{x}), \quad \mathbf{x} \in \Omega_{k}
$$

Edge element interpolation. For any number $\delta>0$, we define the space

$$
H^{\delta}\left(\mathbf{c u r l} ; \Omega_{k}\right)=\left\{\mathbf{v} \in\left(H^{\delta}\left(\Omega_{k}\right)\right)^{3} ; \text { curlv } \in\left(H^{\delta}\left(\Omega_{k}\right)\right)^{3}\right\}
$$

equipped with the norm

$$
\|\mathbf{v}\|_{\delta, \operatorname{curl} ; \Omega_{k}}=\left(\|\mathbf{v}\|_{\delta, \Omega_{k}}^{2}+\|\mathbf{c u r l} \mathbf{v}\|_{\delta, \Omega_{k}}^{2}\right)^{\frac{1}{2}} .
$$

It is known that for any element $K$ in $\mathcal{T}_{h_{k}}$ and an edge $e$ of $K$, the integrals in (2.4) are well-defined (cf. [4]) for any $\mathbf{v} \in X_{p}(K)(p>2)$ given by

$$
X_{p}(K)=\left\{\mathbf{v} \in\left(L^{p}(K)\right)^{3} ; \text { curlv } \in\left(L^{p}(K)\right)^{3}, \mathbf{v} \times \mathbf{n} \in\left(L^{p}(\partial K)\right)^{3}\right\} .
$$


Thus if $\mathbf{v} \in X_{p}(K)$ for any $K \in \mathcal{T}_{h_{k}}$ and $\mathbf{v} \in H\left(\operatorname{curl} ; \Omega_{k}\right)$, we can define its edge element interpolant $\mathbf{r}_{h_{k}} \mathbf{v}$ which is an edge element function in $V_{h_{k}}\left(\Omega_{k}\right)$ and has the same moments as $\mathbf{v}$ on all edges $e \in \mathcal{E}_{h_{k}}$. Also, we can write

$$
\mathbf{r}_{h_{k}} \mathbf{v}(\mathbf{x})=\sum_{e \in \mathcal{E}_{h_{k}}} \lambda_{e}(\mathbf{v}) L_{e}(\mathbf{x}), \quad \mathbf{x} \in \Omega_{k}
$$

and, we can directly verify from above and the Sobolev embedding theorem that $\mathbf{r}_{h_{k}} \mathbf{v}$ is well-defined for any $\mathbf{v} \in H^{\delta}\left(\mathbf{c u r l} ; \Omega_{k}\right)$ with $\delta>\frac{1}{2}$.

Local multiplier spaces on interfaces. In order to define a reasonable edge element space on the global domain $\Omega$, we should choose a suitable (local) multiplier space $W\left(\Gamma_{i j}\right)$ on each face $\Gamma_{i j}$.

Nestedness assumption. Throughout this work we shall consider the case that the triangulations $\mathcal{T}_{h_{i}}$ and $\mathcal{T}_{h_{j}}$ in two neighboring subdomains $\Omega_{i}$ and $\Omega_{j}$ are matching or strictly nested on each face $\Gamma_{i j}$. More accurately, we assume that all faces $\Gamma_{i j}$ can be classified into two groups: Group 1 and Group 2, where for each $\Gamma_{i j}$ in Group $1, \mathcal{T}_{h_{i}}$ and $\mathcal{T}_{h_{j}}$ are strictly nested on $\Gamma_{i j}$, while for each $\Gamma_{i j}$ in Group 2, $\mathcal{T}_{h_{i}}$ and $\mathcal{T}_{h_{j}}$ are matching on $\Gamma_{i j}$ (so $h_{i}=h_{j}$ ).

We shall need the following tangential restrictions of the local subspaces $V_{h_{i}}\left(\Omega_{i}\right)$ and $V_{h_{j}}\left(\Omega_{j}\right)$ on the common face $\Gamma_{i j}$ of $\Omega_{i}$ and $\Omega_{j}$ :

$$
\begin{aligned}
& V_{h_{i}}\left(\Gamma_{i j}\right)=\left\{\mu=\left.(\mathbf{v} \times \mathbf{n})\right|_{\Gamma_{i j}} ; \mathbf{v} \in V_{h_{i}}\left(\Omega_{i}\right)\right\}, \\
& V_{h_{j}}\left(\Gamma_{i j}\right)=\left\{\mu=\left.(\mathbf{v} \times \mathbf{n})\right|_{\Gamma_{i j}} ; \mathbf{v} \in V_{h_{j}}\left(\Omega_{j}\right)\right\} .
\end{aligned}
$$

Actually, one can check that $V_{h_{i}}\left(\Gamma_{i j}\right)$ and $V_{h_{j}}\left(\Gamma_{i j}\right)$ are linear Raviart-Thomas element spaces on $\Gamma_{i j}$.

Now we come to define a local multiplier space $W\left(\Gamma_{i j}\right)$ on each face $\Gamma_{i j}$. We first consider a face $\Gamma_{i j}$ in Group 1. Without loss of generality, we assume that $\mathcal{T}_{h_{i}}$ is coarser than $\mathcal{T}_{h_{j}}$ on $\Gamma_{i j}$, so $h_{i}>h_{j}$. For easy identification, we set $V_{\text {coar }}\left(\Gamma_{i j}\right)=$ $V_{h_{i}}\left(\Gamma_{i j}\right)$ and $V_{\text {fine }}\left(\Gamma_{i j}\right)=V_{h_{j}}\left(\Gamma_{i j}\right)$. Thus $V_{\text {coar }}\left(\Gamma_{i j}\right) \subset V_{\text {fine }}\left(\Gamma_{i j}\right)$. Then we define $W\left(\Gamma_{i j}\right)$ as the following two-dimensional Nedelec element space:

$$
W\left(\Gamma_{i j}\right)=\mathbf{n} \times V_{\text {coar }}\left(\Gamma_{i j}\right)=\left\{\mu=\left.(\mathbf{n} \times \mathbf{v} \times \mathbf{n})\right|_{\Gamma_{i j}} ; \mathbf{v} \in V_{h_{i}}\left(\Omega_{i}\right)\right\} .
$$

We remark that this multiplier space $W\left(\Gamma_{i j}\right)$ can be viewed as a rotation of the Raviart-Thomas space $V_{\text {coar }}\left(\Gamma_{i j}\right)$ by $\pi / 2$.

We then consider a face $\Gamma_{i j}$ in Group 2. Let $\tau$ be the unit normal vector of $\partial \Gamma_{i j}$ such that it is parallel to the face $\Gamma_{i j}$. As we have $h_{i}=h_{j}$ in this case, we set $V\left(\Gamma_{i j}\right)=V_{h_{i}}\left(\Gamma_{i j}\right)=V_{h_{j}}\left(\Gamma_{i j}\right)$. Then we define $W\left(\Gamma_{i j}\right)$ as the following Raviart-Thomas space:

$$
W\left(\Gamma_{i j}\right)=\left\{\mu \in V\left(\Gamma_{i j}\right) ; \mu \cdot \tau=0 \text { on } \partial \Gamma_{i j}\right\} .
$$

We point out that the definitions of the multiplier spaces $W\left(\Gamma_{i j}\right)$ above are very different from that in the existing literature (cf. 7] [21).

\section{Mortar edge Element method}

In this section, we introduce our mortar edge element method and discuss its well-posedness.

From now on, we shall often write $\mathbf{v}_{k}=\left.\mathbf{v}\right|_{\Omega_{k}}$ for any $\mathbf{v} \in L^{2}(\Omega)^{3}$. Let $\mathbf{t}_{i j}$ be the unit tangential vector along the boundary $\partial \Gamma_{i j}$; then we define

$\tilde{V}_{h}(\Omega)=\left\{\mathbf{v} \in\left(L^{2}(\Omega)\right)^{3} ; \mathbf{v}_{k} \in V_{h_{k}}\left(\Omega_{k}\right) \forall k \quad\right.$ and $\quad \mathbf{v}_{i} \cdot \mathbf{t}_{i j}=\mathbf{v}_{j} \cdot \mathbf{t}_{i j}$ on $\left.\partial \Gamma_{i j} \forall \Gamma_{i j}\right\}$. 
The mortar edge element space on the global domain $\Omega$ is then defined by $V_{h}(\Omega)=\left\{\mathbf{v} \in \tilde{V}_{h}(\Omega) ; \quad\right.$ for each $\left.\Gamma_{i j},\left\langle\mathbf{v}_{i} \times \mathbf{n}, \mu\right\rangle_{\Gamma_{i j}}=\left\langle\mathbf{v}_{j} \times \mathbf{n}, \mu\right\rangle_{\Gamma_{i j}} \forall \mu \in W\left(\Gamma_{i j}\right)\right\}$. We emphasize that the space $V_{h}(\Omega)$ is not a subspace of $H(\mathbf{c u r l} ; \Omega)$.

Now we can formulate our edge element approximation to the variational problem (2.3) as follows: Find $\mathbf{u}_{h} \in V_{h}(\Omega)$ such that

$$
\sum_{k=1}^{N} A_{k}\left(\mathbf{u}_{h_{k}}, \mathbf{v}_{h_{k}}\right)=\left(\mathbf{f}, \mathbf{v}_{h}\right), \quad \forall \mathbf{v}_{h} \in V_{h}(\Omega)
$$

where $A_{k}(\cdot, \cdot)$ is defined only on the subdomain $\Omega_{k}$ by

$$
A_{k}\left(\mathbf{u}_{k}, \mathbf{v}_{k}\right)=\left(\alpha \operatorname{curl} \mathbf{u}_{k}, \operatorname{curl} \mathbf{v}_{k}\right)_{\Omega_{k}}+\left(\beta \mathbf{u}_{k}, \mathbf{v}_{k}\right)_{\Omega_{k}}, \quad k=1,2, \cdots, N .
$$

Remark 3.1. For two subdomains $\Omega_{i}$ and $\Omega_{j}$ sharing a common face $\Gamma_{i j}$, let $\mathcal{E}_{i j}$ be the set of all the fine edges of $\mathcal{T}_{h_{i}}$ and $\mathcal{T}_{h_{j}}$ lying on $\partial \Gamma_{i j}$. The condition that $\mathbf{v}_{i} \cdot \mathbf{t}_{i j}=\mathbf{v}_{j} \cdot \mathbf{t}_{i j}$ on $\partial \Gamma_{i j}$ implies that $\lambda_{e}\left(\mathbf{v}_{i}\right)=\lambda_{e}\left(\mathbf{v}_{j}\right)$ for each $e \in \mathcal{E}_{i j}$. This requirement is reasonable since $\mathcal{T}_{h_{i}}$ and $\mathcal{T}_{h_{j}}$ are nested on $\Gamma_{i j}$. But as we shall see, the condition that $\lambda_{e}\left(\mathbf{v}_{i}\right)=\lambda_{e}\left(\mathbf{v}_{j}\right)$ for $e \in \mathcal{E}_{i j}$ is needed only for those faces in Group 2. Such a definition of $V_{h}(\Omega)$ is necessary when designing an efficient solution method for (3.1).

Remark 3.2. If we choose

$$
W\left(\Gamma_{i j}\right)=\left\{\mu \in V_{\text {fine }}\left(\Gamma_{i j}\right) ; \mu \cdot \tau=0 \text { on } \partial \Gamma_{i j}\right\}
$$

for all the faces in Group 1, the same as we did for Group 2, then we have $V_{h}(\Omega) \subset$ $H(\operatorname{curl} ; \Omega)$, see Section 5. But the multiplier space is much larger with such a choice if one of the grids $\mathcal{T}_{h_{i}}$ and $\mathcal{T}_{h_{j}}$ is much finer than the other, and the solution of (3.1) is then much more expensive.

Next, we discuss the unique existence of the mortar edge element system (3.1).

It is easy to see that $V_{h}(\Omega)$ contains some nonzero vector, for example, all the vectors vanishing on the interface $\Gamma$. Moreover, the bilinear form associated with (3.1) is coercive. Thus, we obtain

Theorem 3.1. The mortar edge element problem (3.1) has a unique solution $\mathbf{u}_{h} \in$ $V_{h}(\Omega)$.

It seems difficult to verify the standard inf-sup condition for the system (3.1). Because of this, we will derive in the next section an optimal error estimate of $\mathbf{u}_{h}$ by a novel approach which does not use the standard saddle-point framework (see [7], 8] and [9] for comparison).

We conclude this section with some discussions about the realization of the mortar edge element method (3.1). This can be done basically in two steps.

In the first step, we can establish the algebraic saddle-point system associated with the mortar problem (3.1). For this, we define a product space $W(\Gamma)$ on $\Gamma$ by

$$
W(\Gamma)=\prod_{\Gamma_{i j}} W\left(\Gamma_{i j}\right) .
$$

Then the discrete system (3.1) may be written as the following saddle-point problem: 
Find $\left(\mathbf{u}_{h}, \lambda_{h}\right) \in \tilde{V}_{h}(\Omega) \times W(\Gamma)$ such that

$$
\begin{aligned}
\sum_{k=1}^{N}\left\{A_{k}\left(\mathbf{u}_{h_{k}}, \mathbf{v}_{h_{k}}\right)+\left\langle\mathbf{v}_{h_{k}} \times \mathbf{n}_{k}, \lambda_{h}\right\rangle_{\Gamma_{k}}\right\} & =\left(\mathbf{f}, \mathbf{v}_{h}\right), \quad \forall \mathbf{v}_{h} \in \tilde{V}_{h}(\Omega), \\
\sum_{k=1}^{N}\left\langle\mathbf{u}_{h_{k}} \times \mathbf{n}_{k}, \mu_{h}\right\rangle_{\Gamma_{k}} & =0, \quad \forall \mu_{h} \in W(\Gamma) .
\end{aligned}
$$

Now we discuss how to ensure the solution $\mathbf{u}_{h}$ to meet the edge conditions required in $\tilde{V}_{h}(\Omega)$. Consider a coarse edge $E$ from any one of the subdomains, and let $\Omega_{1}^{\mathrm{E}}, \cdots, \Omega_{k}^{\mathrm{E}}$ denote the subdomains which share $E$ as one of their edges. The triangulation of each subdomain $\Omega_{i}^{\mathrm{E}}$ induces a natural partition of $E$, denoted as $\mathcal{T}_{h_{i}}^{E}(1 \leq i \leq k)$. Without loss of generality, we assume that the partition $\mathcal{T}_{h_{1}}^{E}$ is the coarsest among all the partitions $\mathcal{T}_{h_{1}}^{E}, \cdots, \mathcal{T}_{h_{k}}^{E}$ on $E$, that is, all the partitions $\mathcal{T}_{h_{2}}^{E}$, $\cdots, \mathcal{T}_{h_{k}}^{E}$ are some refinement of $\mathcal{T}_{h_{1}}^{E}$ on $E$. Using (2.6), the restriction of $\mathbf{u}_{h}$ in $\Omega_{1}^{E}$ can be expressed in terms of Nedelec's basis functions associated with the triangulation of $\Omega_{1}^{\mathrm{E}}$. Now, considering an arbitrary fine edge $e \subset E$ from $\mathcal{T}_{h_{1}}^{E}$, let $\lambda_{e}\left(\mathbf{u}_{h}\right)$ be the coefficient of the solution $\left.\mathbf{u}_{h}\right|_{\Omega_{1}^{E}}$ corresponding to Nedelec's basis $L_{e}$ (cf. (2.6)). For any other partition $\mathcal{T}_{h_{i}}^{E}$ with $2 \leq i \leq k$, let $e_{1}^{i}, \cdots, e_{m}^{i}$ denote its fine edges on $e$, and $L_{e_{1}^{i}}, \cdots, L_{e_{m}^{i}}$ be the corresponding Nedelec's basis functions associated with the triangulation of $\Omega_{i}^{E}$. Then one can choose the coefficients $\lambda_{e_{1}^{i}}\left(\mathbf{u}_{h}\right), \cdots, \lambda_{e_{1}^{m}}\left(\mathbf{u}_{h}\right)$ of the solution $\left.\mathbf{u}_{h}\right|_{\Omega_{i}^{E}}(i=2, \cdots, n)$ corresponding to $L_{e_{1}^{i}}, \cdots, L_{e_{m}^{i}}$ as $\lambda_{e}\left(\mathbf{u}_{h}\right) / m$. In other words, if we choose the new basis functions as $L_{e_{1}^{i}} / m, \cdots, L_{e_{m}^{i}} / m$, then the coefficients of the solution $\left.\mathbf{u}_{h}\right|_{\Omega_{i}^{E}}$ on the new basis is also $\lambda_{e}\left(\mathbf{u}_{h}\right)$. Using such basis representations, one can easily verify that the resulting mortar element solution $\mathbf{u}_{h}$ satisfies the edge conditions as required in the space $\tilde{V}_{h}(\Omega)$. With help of this basis representation and the introduction of two appropriate operators $A$ and $B$, the saddle-point system (3.2)-(3.3) can be formulated as follows:

$$
\begin{aligned}
A \mathbf{u}_{h}+B^{t} \lambda_{h} & =0, \\
B \mathbf{u}_{h} & =0 .
\end{aligned}
$$

In the second step, we should work out some effective method to solve the saddlepoint system (3.4)-(3.5). This system can be solved by many existing iterative methods, for example, the inexact Uzawa-type methods developed in [24, 25. But in order for an iterative method to be efficient, one should construct an effective preconditioner $\hat{A}$ for the operator $A$ and another effective preconditioner for the Schur complement $B \hat{A}^{-1} B^{t}$ associated with the system (3.4)-3.5). A mortar edge element method will be of no practical meaning if no effective preconditioners can be found for $A$ and $B \hat{A}^{-1} B^{t}$.

With this aim in mind, the mortar edge element method (3.1) was constructed in a way that effective preconditioners can be found for both $A$ and $B \hat{A}^{-1} B^{t}$. This will be discussed in detail in a separate work.

\section{Generalized edge element interpolation}

We use this section to introduce a generalized interpolation operator which maps any function in $H(\operatorname{curl} ; \Omega)$ and $H^{\delta_{k}}\left(\mathbf{c u r l} ; \Omega_{k}\right)\left(\delta_{k}>\frac{1}{2}\right)$ for all $\Omega_{k}$ 's into an edge element function in $H(\mathbf{c u r l} ; \Omega) \cap V_{h}(\Omega)$ and analyze its interpolation errors. This interpolation operator will play a key role in establishing the error estimates of the 
mortar element method proposed in Section 3 . As we will see, the new interpolation operator is indeed a generalization of the standard one in the edge element space $V_{h}(\Omega)$ when all the subdomain triangulations $\left\{\mathcal{T}_{h_{k}}\right\}$ match with each other on all interfaces, but its definition is rather tricky.

4.1. Helmholtz decomposition and extension operators. We start with the Helmholtz decomposition for edge element functions. Let

$$
H_{0}\left(\operatorname{div}_{0} ; \Omega_{k}\right)=\left\{\mathbf{v} \in H\left(\operatorname{div} ; \Omega_{k}\right) ; \operatorname{div} \mathbf{v}=0 \text { and } \mathbf{v} \cdot \mathbf{n}=0 \text { on } \Gamma_{k}\right\} .
$$

By the Helmholtz decomposition [17, for any $\mathbf{v}_{h_{k}} \in V_{h_{k}}\left(\Omega_{k}\right)$, there exist functions $p \in H^{1}\left(\Omega_{k}\right)$ and $\mathbf{w} \in H\left(\operatorname{curl} ; \Omega_{k}\right) \cap H_{0}\left(\operatorname{div}_{0} ; \Omega_{k}\right)$ such that

$$
\mathbf{v}_{h_{k}}=\nabla p+\mathbf{w} \text {. }
$$

But as $\Omega_{k}$ is a convex polyhedron, we know $\mathbf{w} \in H\left(\right.$ curl; $\left.\Omega_{k}\right) \cap H_{0}\left(\operatorname{div}_{0} ; \Omega_{k}\right) \subset$ $\left(H^{1}\left(\Omega_{k}\right)\right)^{3}$ (cf. [17]). Therefore we have $\mathbf{w} \in\left(H^{1}\left(\Omega_{k}\right)\right)^{3}$, which with the fact that $\operatorname{curl} \mathbf{w}=\mathbf{c u r l} \mathbf{v}_{h_{k}}$ ensures that the interpolant $\mathbf{r}_{h_{k}} \mathbf{w}$ is well-defined; see Section 2 . Taking the interpolation on both sides of (4.1), we see

$$
\mathbf{v}_{h_{k}}=\mathbf{r}_{h_{k}} \nabla p+\mathbf{r}_{h_{k}} \mathbf{w}
$$

Now we introduce a subspace $Z_{h_{k}}\left(\Omega_{k}\right)$ of $H^{1}\left(\Omega_{k}\right)$, which is the continuous piecewise linear nodal finite element space associated with the triangulation $\mathcal{T}_{h_{k}}$. Then one can find (cf. Lemma 5.10, 17]) a function $p_{h_{k}}$ from $Z_{h_{k}}\left(\Omega_{k}\right)$ such that $\mathbf{r}_{h_{k}} \nabla p=\nabla p_{h_{k}}$. With this, we can write

$$
\mathbf{v}_{h_{k}}=\nabla p_{h_{k}}+\mathbf{r}_{h_{k}} \mathbf{w}=\nabla p_{h_{k}}+\mathbf{w}_{h_{k}}
$$

where $\mathbf{w}_{h_{k}}=\mathbf{r}_{h_{k}} \mathbf{w} \in V_{h_{k}}\left(\Omega_{k}\right)$.

From (4.1) and (4.2) we see the following relation:

$$
\operatorname{curl} \mathbf{w}_{h_{k}}=\operatorname{curl} \mathbf{w}=\operatorname{curl} \mathbf{v}_{h_{k}} .
$$

Next, we introduce some additional extension operators.

For any closed subset $G$ of $\Gamma_{k}$, we define

$$
V_{h_{k}}(G)=\left\{\Phi=\left.\mathbf{v}\right|_{G} ; \mathbf{v} \in V_{h_{k}}\left(\Omega_{k}\right)\right\} .
$$

Then we introduce a discrete operator $\mathbf{R}_{G, h_{k}}^{0}$ from $V_{h_{k}}(G)$ into $V_{h_{k}}\left(\Omega_{k}\right)$ such that for any $\Phi=\left.\mathbf{v}\right|_{G}$ with $\mathbf{v} \in V_{h_{k}}\left(\Omega_{k}\right), \mathbf{R}_{G, h_{k}}^{0} \Phi$ is given by

$$
\mathbf{R}_{G, h_{k}}^{0} \Phi(\mathbf{x})=\sum_{e \in \mathcal{E}_{h_{k}} \cap G} \lambda_{e}(\mathbf{v}) L_{e}(\mathbf{x}), \quad \mathbf{x} \in \bar{\Omega}_{k} .
$$

One can easily see that the degree of freedom of $\mathbf{R}_{G, h_{k}}^{0} \Phi$ associated with any edge $e$ outside of $G$ vanishes.

Similarly for the nodal element space $Z_{h_{k}}\left(\Omega_{k}\right)$, we define its restriction on $G$ by

$$
Z_{h_{k}}(G)=\left\{\varphi=\left.v\right|_{G} ; \mathbf{v} \in Z_{h_{k}}\left(\Omega_{k}\right)\right\} .
$$

For any function $\varphi$ in $L^{2}(G)$, let $\gamma_{G}(\varphi)$ be the average value of $\varphi$ on $G$. Consider a closed common edge $\mathrm{E}$ of two subdomains $\Omega_{r}$ and $\Omega_{k}$ and any $\varphi \in Z_{h_{r}}\left(\Gamma_{r}\right)$, we define its extension onto $\Gamma_{k}$ by

$$
\pi_{\mathrm{E}, h_{k}}^{0} \varphi(\mathbf{x})=\left\{\begin{array}{lr}
\varphi(\mathbf{x}) & \text { for } \mathbf{x} \in \mathrm{E} \cap \mathcal{N}_{h_{k}}, \\
\gamma_{\Gamma_{r}}(\varphi) & \text { for } \mathbf{x} \in\left(\Gamma_{k} \backslash \mathrm{E}\right) \cap \mathcal{N}_{h_{k}}
\end{array}\right.
$$

and $\pi_{\mathrm{E}, h_{k}}^{0} \varphi \in Z_{h_{k}}\left(\Gamma_{k}\right)$. 
Finally, we mention that we shall also used the standard discrete harmonic extension $R_{h_{k}}: Z_{h_{k}}\left(\Gamma_{k}\right) \rightarrow Z_{h_{k}}\left(\Omega_{k}\right)$; see [37] for more detail.

4.2. Generalized edge element interpolation operator. With the preparations in the last subsection, we can now introduce a new generalized edge element interpolation operator.

For any $\mathbf{v} \in H(\operatorname{curl} ; \Omega)$ and $H^{\delta_{k}}\left(\operatorname{curl} ; \Omega_{k}\right)$ for each $\Omega_{k}\left(\delta_{k}>\frac{1}{2}\right)$, we will define a new interpolation $\mathbf{r}_{h} \mathbf{v}$ in an appropriate manner so that $\mathbf{r}_{h} \mathbf{v} \in H(\mathbf{c u r l} ; \Omega)$ and $\mathbf{r}_{h} \mathbf{v}$ has some ideal approximation properties. For the sake of exposition, we assume that the grid in each subdomain is uniform. Thus, the subdomains $\Omega_{1}, \Omega_{2}, \cdots, \Omega_{N}$ can be arranged in such an order that $h_{1} \geq h_{2} \geq \cdots \geq h_{N}$. For the general case with quasi-uniform grids, some obvious modifications of the subsequent definition of the generalized interpolation $\mathbf{r}_{h} \mathbf{v}$ are needed. We shall first define $\mathbf{r}_{h} \mathbf{v}$ on all interfaces (common edges and faces), and then extend the definition into all subdomains as it is done in the following.

In the sequel we shall write $\mathbf{v}_{k}=\left.\mathbf{v}\right|_{\Omega_{k}}$ and $\mathbf{v}_{h_{k}}=\left.\left(\mathbf{r}_{h} \mathbf{v}\right)\right|_{\Omega_{k}}$.

Step 1. The definition of $\mathbf{v}_{h_{1}}$ is as the standard:

$$
\mathbf{v}_{h_{1}}=\mathbf{r}_{h_{1}} \mathbf{v}_{1}=\sum_{e \in \mathcal{E}_{h_{1}}} \lambda_{e}\left(\mathbf{v}_{1}\right) L_{e} \quad \text { in } \quad \Omega_{1} .
$$

Step 2. $\mathbf{v}_{h_{2}}$ is defined by means of $\mathbf{v}_{h_{1}}$.

In the case that $\Gamma_{1} \cap \Gamma_{2}=\emptyset$ or the triangulations $\mathcal{T}_{h_{1}}$ and $\mathcal{T}_{h_{2}}$ are matching on $\Gamma_{12}$, we define $\mathbf{v}_{h_{2}}$ also as the standard:

$$
\mathbf{v}_{h_{2}}=\mathbf{r}_{h_{2}} \mathbf{v}_{2}=\sum_{e \in \mathcal{E}_{h_{2}}} \lambda_{e}\left(\mathbf{v}_{2}\right) L_{e} \quad \text { in } \quad \Omega_{2} .
$$

Otherwise we have to define $\mathbf{v}_{h_{2}}$ very carefully. There are two different situations: (i) $\Gamma_{1} \cap \Gamma_{2}=\Gamma_{12}$ is a face; (ii) $\Gamma_{1} \cap \Gamma_{2}=\mathrm{E}_{12}$ is an edge. For case (i), the treatment is simple; but case (ii) is more tricky.

For the case (i), we define

$$
\mathbf{v}_{h_{2}}=\mathbf{r}_{h_{2}} \mathbf{v}_{2}+\mathbf{R}_{\Gamma_{12}, h_{2}}^{0}\left[\left.\left(\mathbf{r}_{h_{1}} \mathbf{v}_{1}-\mathbf{r}_{h_{2}} \mathbf{v}_{2}\right)\right|_{\Gamma_{12}}\right]
$$

Now we treat case (ii). Recall that $\mathcal{T}_{12}$ and $\mathcal{T}_{21}$ are the respective restrictions of $\mathcal{T}_{h_{1}}$ and $\mathcal{T}_{h_{2}}$ on $\Gamma_{12}$. We then extend $\mathcal{T}_{12}$ and $\mathcal{T}_{21}$ both into $\Omega_{1}$ with mesh sizes $h_{1}$ and $h_{2}$, and the extended triangulations are denoted by $\tilde{\mathcal{T}}_{12}$ and $\tilde{\mathcal{T}}_{21}$, respectively. Since $\mathcal{T}_{12}$ and $\mathcal{T}_{21}$ are nested, we require that $\tilde{\mathcal{T}}_{12}$ and $\tilde{\mathcal{T}}_{21}$ are also nested. Let $\tilde{\mathbf{r}}_{h_{1}}$ and $\tilde{\mathbf{r}}_{h_{2}}$ denote the interpolation operators associated with $\tilde{\mathcal{T}}_{12}$ and $\tilde{\mathcal{T}}_{21}$, respectively.

The same as in (4.1), we can have the Helmholtz decomposition for $\tilde{\mathbf{r}}_{h_{1}} \mathbf{v}_{1}-\tilde{\mathbf{r}}_{h_{2}} \mathbf{v}_{1}$ :

$$
\tilde{\mathbf{r}}_{h_{1}} \mathbf{v}_{1}-\tilde{\mathbf{r}}_{h_{2}} \mathbf{v}_{1}=\mathbf{w}+\nabla p
$$

with $\mathbf{w} \in H\left(\operatorname{curl} ; \Omega_{1}\right) \cap H_{0}\left(\operatorname{div}_{0} ; \Omega_{1}\right)$ and $p \in H^{1}\left(\Omega_{1}\right)$. Applying $\tilde{\mathbf{r}}_{h_{2}}$, we have

$$
\tilde{\mathbf{r}}_{h_{1}} \mathbf{v}_{1}-\tilde{\mathbf{r}}_{h_{2}} \mathbf{v}_{1}=\tilde{\mathbf{r}}_{h_{2}} \mathbf{w}+\tilde{\mathbf{r}}_{h_{2}} \nabla p=\tilde{\mathbf{w}}_{h_{2}}+\nabla \tilde{p}_{h_{2}},
$$

where $\tilde{\mathbf{w}}_{h_{2}}=\tilde{\mathbf{r}}_{h_{2}} \mathbf{w}$ and $\tilde{p}_{h_{2}} \in H^{1}\left(\Omega_{1}\right)$ is a piecewise linear function associated with the extended triangulation $\tilde{\mathcal{T}}_{21}$ of $\Omega_{1}$. Then we define

$$
\mathbf{v}_{h_{k}}=\mathbf{r}_{h_{2}} \mathbf{v}_{2}+\mathbf{R}_{\mathrm{E}_{12}, h_{2}}^{0}\left(\left.\tilde{\mathbf{w}}_{h_{2}}\right|_{\mathrm{E}_{12}}\right)+\nabla p_{h_{2}}
$$

where $p_{h_{2}} \in H^{1}\left(\Omega_{2}\right)$ is a discrete harmonic function in $\Omega_{2}$ given by

$$
p_{h_{2}}=R_{h_{2}}\left[\pi_{\mathrm{E}_{12}, h_{2}}^{0}\left(\left.\tilde{p}_{h_{2}}\right|_{\Gamma_{12}}\right)\right] .
$$

Step 3. Assume that $\mathbf{v}_{h_{1}}, \mathbf{v}_{h_{2}}, \cdots, \mathbf{v}_{h_{k-1}}$ are defined. We define $\mathbf{v}_{h_{k}}$ below. 
In the case that $\Gamma_{r} \cap \Gamma_{k}=\emptyset$ for $r=1, \cdots, k-1$ or triangulations $\mathcal{T}_{h_{k}}$ and $\mathcal{T}_{h_{r}}$ matches on $\Gamma_{r k}$ for some $1 \leq r \leq k-1$, then we define $\mathbf{v}_{h_{k}}$ as the standard interpolation:

$$
\mathbf{v}_{h_{k}}=\mathbf{r}_{h_{k}} \mathbf{v}_{k}
$$

Otherwise, let $\Omega_{k_{1}}, \cdots, \Omega_{k_{n}}$ with $k_{1}<\cdots<k_{n}<k$ be the subdomains such that $\Gamma_{k_{l}} \cap \Gamma_{k} \neq \emptyset(l=1, \cdots, n)$. Without loss of generality, we assume that $\Gamma_{k_{l}} \cap \Gamma_{k}=\Gamma_{k_{l} k}$ is a face $(l=1, \cdots, n-1)$, and $\Gamma_{k_{n}} \cap \Gamma_{k}=\mathrm{E}_{k_{n} k}$ is an edge. The face case can be treated in the same manner as in (4.5). To deal with the edge $\mathrm{E}_{k_{n} k}$, we have to consider two cases: (a) there exists one subdomain among all $\Omega_{k_{l}}$ 's $(l<n)$ such that $\mathrm{E}_{k_{n} k}$ is also one of its edges; (b) $\mathrm{E}_{k_{n} k}$ is not an edge of any subdomain from $\Omega_{k_{l}}(l<n)$. For case (a), we can define $\mathbf{v}_{h_{k}}$ directly:

$$
\mathbf{v}_{h_{k}}=\mathbf{r}_{h_{k}} \mathbf{v}_{k}+\sum_{l=1}^{n-1} \mathbf{R}_{\Gamma_{k_{l} k}, h_{k}}^{0}\left[\left.\left(\mathbf{r}_{h_{k_{l}}} \mathbf{v}_{k_{l}}-\mathbf{r}_{h_{k}} \mathbf{v}_{k}\right)\right|_{\Gamma_{k_{l} k}}\right]
$$

The case (b) is more complicated. As we did in case (ii) of Step 2, we define two auxiliary interpolation operators $\tilde{\mathbf{r}}_{h_{k_{n}}}$ and $\tilde{\mathbf{r}}_{h_{k}}$ in $\Omega_{k_{n}}$, and let $\tilde{\mathbf{w}}_{h_{k}}$ and $\tilde{p}_{h_{k}}$ be obtained by Helmholtz decomposition of $\tilde{\mathbf{r}}_{h_{k_{n}}} \mathbf{v}_{k_{n}}-\tilde{\mathbf{r}}_{h_{k}} \mathbf{v}_{k}$ as in (4.7). Then we define

$\mathbf{v}_{h_{k}}=\mathbf{r}_{h_{k}} \mathbf{v}_{k}+\sum_{l=1}^{n-1} \mathbf{R}_{\Gamma_{k_{l} k}, h_{k}}^{0}\left[\left.\left(\mathbf{r}_{h_{k_{l}}} \mathbf{v}_{k_{l}}-\mathbf{r}_{h_{k}} \mathbf{v}_{k}\right)\right|_{\Gamma_{k_{l} k}}\right]+\mathbf{R}_{\mathrm{E}_{k_{n} k}, h_{k}}^{0}\left(\left.\tilde{\mathbf{w}}_{h_{k}}\right|_{\mathrm{E}_{k_{n} k}}\right)+\nabla p_{h_{k}}$

where $p_{h_{k}}$ is discrete harmonic in $\Omega_{k}$ and given by

$$
p_{h_{k}}=R_{h_{k}}\left[\pi_{\mathrm{E}_{k_{n} k}, h_{k}}^{0}\left(\left.\tilde{p}_{h_{k}}\right|_{\Gamma_{k_{n}}}\right)\right] \text {. }
$$

With the previously defined $\mathbf{v}_{h_{i}}, i=1,2, \cdots, N$, we can now define the generalized interpolation operator $\mathbf{r}_{h} \mathbf{v}$ simply by $\mathbf{r}_{h} \mathbf{v}=\mathbf{v}_{h_{i}}$ in each subdomain $\Omega_{i}$.

4.3. Interpolation error estimates. In this subsection we shall establish the error estimates for the generalized interpolation operator $\mathbf{r}_{h}$ defined earlier. We start with the justification that $\mathbf{r}_{h}$ is indeed a generalized edge element interpolation operator.

Lemma 4.1. If the subdomain triangulations $\left\{\mathcal{T}_{h_{k}}\right\}$ are matching on all faces $\Gamma_{i j}$ of each subdomain, then the generalized operator $\mathbf{r}_{h}$ reduces to the standard edge element interpolation in the whole domain $\Omega$.

Proof. For any $\mathbf{v} \in H(\mathbf{c u r l} ; \Omega)$ and $H^{\delta_{k}}\left(\mathbf{c u r l} ; \Omega_{k}\right)$ for each $\Omega_{k}\left(1 / 2<\delta_{k} \leq 1\right)$, by the definition of the generalized interpolation operator $\mathbf{r}_{h}$ it suffices to verify that for each fixed $k, 1<k \leq N$, we have $\mathbf{r}_{h_{k_{l}}} \mathbf{v}_{k_{l}}=\mathbf{r}_{h_{k}} \mathbf{v}_{k}$ on $\Gamma_{k_{l} k}$ for all $k_{l}$ such that $1 \leq k_{l}<k$.

If the grids $\left\{\mathcal{T}_{h_{k}}\right\}$ are matching on all faces $\Gamma_{i j}$, we have $\mathbf{r}_{h_{k_{l}}}=\mathbf{r}_{h_{k}}$ on $\Gamma_{k_{l} k}$. Thus we need only to check

$$
\lambda_{e}\left(\mathbf{r}_{h_{k}} \mathbf{v}_{k_{l}}\right)=\lambda_{e}\left(\mathbf{r}_{h_{k}} \mathbf{v}_{k}\right) \quad \forall e \in \mathcal{E}_{h_{k}} \cap \Gamma_{k_{l} k} .
$$

As $\mathbf{v} \in H(\mathbf{c u r l} ; \Omega) \cap \prod_{k=1}^{N} H^{\delta_{k}}\left(\mathbf{c u r l} ; \Omega_{k}\right)$ with $1 / 2<\delta_{k} \leq 1$, the moments $\lambda_{e}(\mathbf{v})$ are well-defined for all edges $e$ on $\Gamma_{k_{l} k}$; see [2], [15]. In particular, we have $\lambda_{e}\left(\mathbf{v}_{k_{l}}\right)=$ $\lambda_{e}\left(\mathbf{v}_{k}\right)$ for all $e$ on $\Gamma_{k_{l} k}$ (note that $\left.\Gamma_{k_{l} k} \neq \emptyset\right)$. Now by the definition of $\mathbf{r}_{h_{k}}$, we see

$$
\lambda_{e}\left(\mathbf{r}_{h_{k}} \mathbf{v}_{k_{l}}\right)=\lambda_{e}\left(\mathbf{v}_{k_{l}}\right)=\lambda_{e}\left(\mathbf{v}_{k}\right)=\lambda_{e}\left(\mathbf{r}_{h_{k}} \mathbf{v}_{k}\right),
$$

which gives (4.8). 
Lemma 4.2. For any $\mathbf{v} \in H(\operatorname{curl} ; \Omega) \cap \prod_{k=1}^{N} H^{\delta_{k}}\left(\operatorname{curl} ; \Omega_{k}\right)$ with $1 / 2<\delta_{k} \leq 1$, we have

$$
\mathbf{r}_{h} \mathbf{v} \in H(\operatorname{curl} ; \Omega) \cap V_{h}(\Omega) \quad \text { and }\left.\quad\left(\mathbf{r}_{h} \mathbf{v} \times \mathbf{n}\right)\right|_{\Gamma_{i j}} \in V_{\text {coar }}\left(\Gamma_{i j}\right) \quad \forall \Gamma_{i j} .
$$

Proof. It is clear that $\left.\left(\mathbf{r}_{h} \mathbf{v}\right)\right|_{\Omega_{k}} \in V_{h_{k}}\left(\Omega_{k}\right)$ for every subdomain $\Omega_{k}$. To see $\mathbf{r}_{h} \mathbf{v} \in$ $H(\operatorname{curl} ; \Omega)$, we have to verify the tangential continuity condition: $\left.\left(\mathbf{r}_{h} \mathbf{v}\right)\right|_{\Omega_{i}} \times \mathbf{n}=$ $\left.\left(\mathbf{r}_{h} \mathbf{v}\right)\right|_{\Omega_{j}} \times \mathbf{n}$ on $\Gamma_{i j}$. By the definition of $\mathbf{r}_{h}$, this is equivalent to the condition $\mathbf{v}_{h_{i}} \times \mathbf{n}=\mathbf{v}_{h_{j}} \times \mathbf{n}$ on $\Gamma_{i j}$ for each face $\Gamma_{i j}$. Without loss of generality, we assume that $i<j$ and $h_{i}>h_{j}$. Since $\mathcal{T}_{h_{i}}$ and $\mathcal{T}_{h_{j}}$ are nested on $\Gamma_{i j}$, we have $V_{h_{i}}\left(\Gamma_{i j}\right) \subset V_{h_{j}}\left(\Gamma_{i j}\right)$. It follows, by the definition of $\mathbf{v}_{h_{k}}$, that $\lambda_{e}\left(\mathbf{v}_{h_{j}}\right)=\lambda_{e}\left(\mathbf{v}_{h_{i}}\right)$ for any $e \subset \Gamma_{i j} \cap \mathcal{T}_{h_{j}}$. Thus $\mathbf{v}_{h_{j}} \times \mathbf{n}=\mathbf{v}_{h_{i}} \times \mathbf{n}$ on $K$ for each (fine) element $K$ of $\mathcal{T}_{h_{j}}$ on $\Gamma_{i j}$, which implies that $\mathbf{v}_{h_{i}} \times \mathbf{n}=\mathbf{v}_{h_{j}} \times \mathbf{n}$ on $\Gamma_{i j}$, so we know that $\left.\left(\mathbf{v}_{h_{j}} \times \mathbf{n}\right)\right|_{\Gamma_{i j}}$ lies in the space $V_{h_{i}}\left(\Gamma_{i j}\right)=V_{\text {coar }}\left(\Gamma_{i j}\right)$.

In the remaining part of this section, we will derive the interpolation error estimates for the generalized interpolation operator $\mathbf{r}_{h}$.

For simplicity, we will frequently use the notations $\lesssim$ and $\underset{\sim}{\sim}$. For any two nonnegative quantities $x$ and $y, x \lesssim y$ means that $x \leq C y$ for some constant $C$ independent of mesh size $h$, subdomain size $d$ and the related parameters. $x \underset{\approx}{\sim}$ means $x \lesssim y$ and $y \lesssim x$.

We start with the introduction of a few auxiliary results.

First, we recall that in each subdomain $\Omega_{k}, \mathbf{r}_{h_{k}}$ is the standard edge element interpolation associated with the space $V_{h_{k}}\left(\Omega_{k}\right)$, so the interpolation error estimates in the next lemma are well-known (cf. [2] [15]):

Lemma 4.3. For any $\mathbf{v} \in H^{\delta_{k}}\left(\operatorname{curl} ; \Omega_{k}\right)$ with $\delta_{k}>\frac{1}{2}$, we have

$$
\left\|\mathbf{r}_{h_{k}} \mathbf{v}-\mathbf{v}\right\|_{\mathbf{c u r l}, \Omega_{k}} \lesssim h_{k}^{\delta}\|\operatorname{curlv}\|_{\delta, \Omega_{k}} .
$$

The following Lemma 4.4 can be shown basically in the same manner as the proof of Lemma 4.5 in [26, while Lemma 4.5 can be found in [26].

Lemma 4.4. Let $\mathrm{E}_{k}$ be an edge of $\Omega_{k}$. For any $\mathbf{w} \in H\left(\operatorname{curl} ; \Omega_{k}\right) \cap H_{0}\left(\operatorname{div}_{0} ; \Omega_{k}\right)$, if its interpolant $\mathbf{r}_{h_{k}} \mathbf{w}$ is well-defined in $V_{h_{k}}\left(\Omega_{k}\right)$, then we have

$$
\left\|\mathbf{r}_{h_{k}} \mathbf{w}\right\|_{0, \mathrm{E}_{k}} \lesssim\left[1+\log \left(d / h_{k}\right)\right]^{\frac{1}{2}}\|\mathbf{c u r l w}\|_{0, \Omega_{k}} .
$$

Lemma 4.5. Let $\mathbf{v}_{h_{k}} \in V_{h_{k}}\left(\Omega_{k}\right)$, and $p_{h_{k}} \in Z_{h_{k}}\left(\Omega_{k}\right)$ be defined by the Helmholtz decomposition as in (4.2). Then

$$
\left|p_{h_{k}}\right|_{1, \Omega_{k}} \lesssim\left\|\mathbf{v}_{h_{k}}\right\|_{\mathbf{c u r l}, \Omega_{k}} .
$$

Lemma 4.6. Let $\Omega_{r}$ and $\Omega_{k}$ be two subdomains sharing a common edge E. Then for any $\varphi \in Z_{h_{r}}\left(\Gamma_{r}\right)$ we have

$$
\left|\pi_{\mathrm{E}, h_{k}}^{0} \varphi\right|_{\frac{1}{2}, \Gamma_{k}} \lesssim\left[1+\log \left(d / h_{r}\right)\right]^{\frac{1}{2}}|\varphi|_{\frac{1}{2}, \Gamma_{r}} .
$$

Proof. Using the inverse inequality,

$$
\left|\pi_{\mathrm{E}, h_{k}}^{0} \varphi\right|_{\frac{1}{2}, \Gamma_{k}}=\left|\pi_{\mathrm{E}, h_{k}}^{0} \varphi-\gamma_{\Gamma_{r}}(\varphi)\right|_{\frac{1}{2}, \Gamma_{k}} \lesssim h_{k}^{-\frac{1}{2}}\left\|\pi_{\mathrm{E}, h_{k}}^{0} \varphi-\gamma_{\Gamma_{r}}(\varphi)\right\|_{0, \Gamma_{k}} .
$$


By the definition of $\pi_{\mathrm{E}, h_{k}}^{0}$, we know $\pi_{\mathrm{E}, h_{k}}^{0} \varphi-\gamma_{\Gamma_{r}}(\varphi) \in Z_{h_{k}}\left(\Gamma_{k}\right)$ and can be viewed as the zero extension of $\left.\left(\varphi-\gamma_{\Gamma_{r}}(\varphi)\right)\right|_{\mathrm{E}}$ on to the entire boundary $\Gamma_{k}$. Then it is easy to see that

$$
\left\|\pi_{\mathrm{E}, h_{k}}^{0} \varphi-\gamma_{\Gamma_{r}}(\varphi)\right\|_{0, \Gamma_{k}} \lesssim h_{k}^{\frac{1}{2}}\left\|\varphi-\gamma_{\Gamma_{r}}(\varphi)\right\|_{0, \mathrm{E}}
$$

Plugging this in (4.13), and using Lemma 4.9 in [37, leads to

$$
\left|\pi_{\mathrm{E}, h_{k}}^{0} \varphi\right|_{\frac{1}{2}, \Gamma_{k}} \lesssim\left[1+\log \left(d / h_{k}\right)\right]^{\frac{1}{2}}\left\|\varphi-\gamma_{\Gamma_{r}}(\varphi)\right\|_{\frac{1}{2}, \Gamma_{r}} .
$$

Then the desired estimate (4.12) follows from Friedrich's inequality.

Finally we are ready to establish the interpolation error estimates for the generalized interpolation operator $\mathbf{r}_{h}$.

Theorem 4.1. Let $\mathbf{r}_{h}$ be the generalized interpolation operator defined in Section 4.2. Then for any $\mathbf{v} \in H(\operatorname{curl} ; \Omega) \cap \prod_{k=1}^{N} H^{\delta_{k}}\left(\operatorname{curl} ; \Omega_{k}\right)$ with $1 / 2<\delta_{k} \leq 1$, we have

$$
\left\|\mathbf{r}_{h} \mathbf{v}-\mathbf{v}\right\|_{\mathbf{c u r l}, \Omega} \lesssim[1+\log (d / h)]^{\frac{1}{2}}\left(\sum_{k=1}^{N} h_{k}^{2 \delta_{k}}\|\operatorname{curl} \mathbf{v}\|_{\delta_{k}, \Omega_{k}}^{2}\right)^{\frac{1}{2}} .
$$

Proof. It follows from Lemma 4.3 that

$$
\left\|\mathbf{r}_{h} \mathbf{v}-\mathbf{v}\right\|_{\mathbf{c u r l}, \Omega_{1}}=\left\|\mathbf{r}_{h_{1}} \mathbf{v}-\mathbf{v}_{1}\right\|_{\operatorname{curl}, \Omega_{1}} \lesssim h_{1}^{\delta_{1}}\|\mathbf{c u r l} \mathbf{v}\|_{\delta_{1}, \Omega_{1}} .
$$

Next we prove that

$$
\left\|\mathbf{r}_{h} \mathbf{v}-\mathbf{v}\right\|_{\operatorname{curl}, \Omega_{2}} \lesssim h_{1}^{\delta_{1}}\|\mathbf{c u r l} \mathbf{v}\|_{\delta_{1}, \Omega_{1}}+h_{2}^{\delta_{2}}\|\operatorname{curl} \mathbf{v}\|_{\delta_{2}, \Omega_{2}} .
$$

This follows immediately from Lemma 4.3 for the case that $\partial \Omega_{1} \cap \partial \Omega_{2}=\emptyset$. Below, we will consider the case that $\partial \Omega_{1} \cap \partial \Omega_{2} \neq \emptyset$. This proof is divided into three steps.

Step 1. Estimate $\left\|\mathbf{r}_{h} \mathbf{v}-\mathbf{v}\right\|_{0, \Omega_{2}}$ when $\partial \Omega_{1} \cap \partial \Omega_{2}=\Gamma_{12}$ is a face.

By the definition of $\mathbf{v}_{h_{2}}$, Lemma 4.3 and the fact that each $L_{e}$ has a small support, we derive

$$
\begin{aligned}
\left\|\mathbf{v}_{h_{2}}-\mathbf{v}_{2}\right\|_{0, \Omega_{2}}^{2} & \lesssim\left\|\mathbf{r}_{h_{2}} \mathbf{v}_{2}-\mathbf{v}_{2}\right\|_{0, \Omega_{2}}^{2}+\left\|\mathbf{R}_{\Gamma_{12}, h_{2}}^{0}\left[\left.\left(\mathbf{r}_{h_{1}} \mathbf{v}_{1}-\mathbf{r}_{h_{2}} \mathbf{v}_{2}\right)\right|_{\Gamma_{12}}\right]\right\|_{0, \Omega_{2}}^{2} \\
& \lesssim h_{2}^{2 \delta_{2}}\left\|\operatorname{curl}_{2}\right\|_{\delta_{2}, \Omega_{2}}+\sum_{e \in \mathcal{E}_{h_{2}} \cap \Gamma_{12}}\left|\lambda_{e}\left(\mathbf{r}_{h_{1}} \mathbf{v}_{1}-\mathbf{r}_{h_{2}} \mathbf{v}_{2}\right)\right|^{2}\left\|L_{e}\right\|_{0, \Omega_{2}}^{2} .
\end{aligned}
$$

One can see from the proof of Lemma 4.1 that

$$
\lambda_{e}\left(\mathbf{r}_{h_{2}} \mathbf{v}_{2}\right)=\lambda_{e}\left(\mathbf{r}_{h_{2}} \mathbf{v}_{1}\right), \quad \forall e \subset \Gamma_{12} \cap \mathcal{T}_{h_{2}} .
$$

So we deduce

$$
\left\|\mathbf{v}_{h_{2}}-\mathbf{v}_{2}\right\|_{0, \Omega_{2}}^{2} \lesssim h_{2}^{2 \delta_{2}}\left\|\operatorname{curl}_{2}\right\|_{\delta_{2}, \Omega_{2}}+\sum_{e \in \mathcal{E}_{h_{2}} \cap \Gamma_{12}}\left|\lambda_{e}\left(\mathbf{r}_{h_{1}} \mathbf{v}_{1}-\mathbf{r}_{h_{2}} \mathbf{v}_{1}\right)\right|^{2}\left\|L_{e}\right\|_{0, \Omega_{2}}^{2} .
$$

But by direct computations, one obtains

$$
\left.\left\|L_{e}\right\|_{0, \Omega_{2}}^{2} \lesssim h_{2}, \quad\left|\lambda_{e}\left(\mathbf{r}_{h_{1}} \mathbf{v}_{1}-\mathbf{r}_{h_{2}} \mathbf{v}_{1}\right)\right|^{2} \lesssim h_{2} \| \mathbf{r}_{h_{1}} \mathbf{v}_{1}-\mathbf{r}_{h_{2}} \mathbf{v}_{1}\right) \|_{0, e}^{2}
$$

Substituting these into (4.18) gives

$$
\left\|\mathbf{v}_{h_{2}}-\mathbf{v}_{2}\right\|_{0, \Omega_{2}}^{2} \lesssim h_{2}^{2 \delta_{2}}\left\|\operatorname{curl}_{2}\right\|_{\delta_{2}, \Omega_{2}}+h_{2}^{2} \sum_{e \in \mathcal{E}_{h_{2}} \cap \Gamma_{12}}\left\|\mathbf{r}_{h_{1}} \mathbf{v}_{1}-\mathbf{r}_{h_{2}} \mathbf{v}_{1}\right\|_{0, e}^{2}
$$


Now let $K_{e}$ be a fine element in $\Omega_{1}$ which has $e$ as one of its edges. As in the proof of Lemma 4.5 in [26, one can verify that

$$
\left\|\mathbf{r}_{h_{1}} \mathbf{v}_{1}-\mathbf{r}_{h_{2}} \mathbf{v}_{1}\right\|_{0, e}^{2}=\left\|\tilde{\mathbf{r}}_{h_{1}} \mathbf{v}_{1}-\tilde{\mathbf{r}}_{h_{2}} \mathbf{v}_{1}\right\|_{0, e}^{2} \lesssim h_{2}^{-2}\left\|\tilde{\mathbf{r}}_{h_{1}} \mathbf{v}_{1}-\tilde{\mathbf{r}}_{h_{2}} \mathbf{v}_{1}\right\|_{0, K_{e}}^{2} .
$$

Using this and Lemma 4.3 we obtain

$$
\begin{aligned}
h_{2}^{2} \sum_{e \in \mathcal{E}_{h_{2}} \cap \Gamma_{12}}\left\|\mathbf{r}_{h_{1}} \mathbf{v}_{1}-\mathbf{r}_{h_{2}} \mathbf{v}_{1}\right\|_{0, e}^{2} & \lesssim \sum_{e \in \in \mathcal{E}_{h_{2}} \cap \Gamma_{12}}\left\|\tilde{\mathbf{r}}_{h_{1}} \mathbf{v}_{1}-\tilde{\mathbf{r}}_{h_{2}} \mathbf{v}_{1}\right\|_{0, K_{e}}^{2} \\
& \lesssim\left\|\tilde{\mathbf{r}}_{h_{1}} \mathbf{v}_{1}-\tilde{\mathbf{r}}_{h_{2}} \mathbf{v}_{1}\right\|_{0, \Omega_{1}}^{2} \\
& \lesssim\left\|\tilde{\mathbf{r}}_{h_{1}} \mathbf{v}_{1}-\mathbf{v}_{1}\right\|_{0, \Omega_{1}}^{2}+\left\|\mathbf{v}_{1}-\tilde{\mathbf{r}}_{h_{2}} \mathbf{v}_{1}\right\|_{0, \Omega_{1}}^{2} \\
& \lesssim h_{1}^{2 \delta_{1}}\left\|\operatorname{currl}_{1}\right\|_{\delta_{1}, \Omega_{1}}^{2}+h_{2}^{2 \delta_{1}}\left\|\operatorname{curl}_{1}\right\|_{\delta_{1}, \Omega_{1}}^{2} \\
& \lesssim h_{1}^{2 \delta_{1}}\left\|\operatorname{currl}_{1}\right\|_{\delta_{1}, \Omega_{1}}^{2} .
\end{aligned}
$$

This, together with (4.19), gives

$$
\left\|\mathbf{v}_{h_{2}}-\mathbf{v}_{2}\right\|_{0, \Omega_{2}}^{2} \lesssim h_{1}^{2 \delta_{1}}\left\|\operatorname{curl}_{1}\right\|_{\delta_{1}, \Omega_{1}}^{2}+h_{2}^{2 \delta_{2}}\left\|\operatorname{curl}_{2}\right\|_{\delta_{2}, \Omega_{2}}^{2}
$$

Step 2. Estimate $\left\|\operatorname{curl}\left(\mathbf{r}_{h} \mathbf{v}-\mathbf{v}\right)\right\|_{0, \Omega_{2}}$ when $\partial \Omega_{1} \cap \partial \Omega_{2}=\Gamma_{12}$ is a face.

By the definition of $\mathbf{v}_{h_{2}}$ and (4.17), we can write

$$
\operatorname{curl}\left(\mathbf{v}_{h_{2}}-\mathbf{v}_{2}\right)=\operatorname{curl}\left(\mathbf{r}_{h_{2}} \mathbf{v}_{2}-\mathbf{v}_{2}\right)+\sum_{e \subset \in \mathcal{E}_{h_{2}} \cap \Gamma_{12}} \lambda_{e}\left(\mathbf{r}_{h_{1}} \mathbf{v}_{1}-\mathbf{r}_{h_{2}} \mathbf{v}_{1}\right) \operatorname{curl} L_{e} .
$$

Recalling that $\mathcal{T}_{21}$ is the restriction of the fine triangulation $\mathcal{T}_{h_{2}}$ on $\Gamma_{12}$, we have

$$
\sum_{e \in \mathcal{E}_{h_{2}} \cap \Gamma_{12}} \lambda_{e}\left(\mathbf{r}_{h_{1}} \mathbf{v}_{1}-\mathbf{r}_{h_{2}} \mathbf{v}_{1}\right) \operatorname{curl} L_{e}=\frac{1}{2} \sum_{K \subset \mathcal{T}_{21}} \sum_{e \subset K} \lambda_{e}\left(\mathbf{r}_{h_{1}} \mathbf{v}_{1}-\mathbf{r}_{h_{2}} \mathbf{v}_{1}\right) \operatorname{curl} L_{e} .
$$

As shown in the proof of Lemma 5.4 in [3], we know

$$
\sum_{e \subset K} \lambda_{e}\left(\mathbf{r}_{h_{1}} \mathbf{v}_{1}-\mathbf{r}_{h_{2}} \mathbf{v}_{1}\right) \operatorname{curl} L_{e}=\mathbf{C}_{K} h_{2}^{-2} \int_{K} \operatorname{curl}\left(\mathbf{r}_{h_{1}} \mathbf{v}_{1}-\mathbf{r}_{h_{2}} \mathbf{v}_{1}\right) \cdot \mathbf{n} d s,
$$

where $\mathbf{C}_{K}$ is a constant vector independent $h_{2}$. Using this relation and the CauchySchwarz inequality, we derive from (4.22) that

$$
\begin{aligned}
& \left\|\sum_{e \in \mathcal{E}_{h_{2}} \cap \Gamma_{12}} \lambda_{e}\left(\mathbf{r}_{h_{1}} \mathbf{v}_{1}-\mathbf{r}_{h_{2}} \mathbf{v}_{1}\right) \operatorname{curl} L_{e}\right\|_{0, \Omega_{2}}^{2} \\
\lesssim & \left.\sum_{K \subset \mathcal{T}_{21}} \| \mathbf{C}_{K} h_{2}^{-2} \int_{K} \operatorname{curlr}_{h_{1}} \mathbf{v}_{1}-\mathbf{r}_{h_{2}} \mathbf{v}_{1}\right) \cdot \mathbf{n} d s \|_{0, \Omega_{2}}^{2} \\
\lesssim & h_{2}^{-1} \sum_{K \subset \mathcal{T}_{21}}\left|\int_{K} \operatorname{curl}\left(\mathbf{r}_{h_{1}} \mathbf{v}_{1}-\mathbf{r}_{h_{2}} \mathbf{v}_{1}\right) \cdot \mathbf{n} d s\right|^{2} \\
\lesssim & h_{2} \sum_{K \subset \mathcal{T}_{21}}\left\|\operatorname{curl}\left(\mathbf{r}_{h_{1}} \mathbf{v}_{1}-\mathbf{r}_{h_{2}} \mathbf{v}_{1}\right)\right\|_{0, K}^{2} .
\end{aligned}
$$

Now we extend $\mathcal{T}_{21}$ into $\Omega_{1}$ to generate an auxiliary quasi-uniform triangulation $\tilde{\mathcal{T}}_{21}$ of $\Omega_{1}$ with mesh size $h_{2}$, and let $\tau_{K}$ be an element in $\tilde{\mathcal{T}}_{21}$ with $K$ as one of its faces. One can verify that

$$
\left\|\operatorname{curl}\left(\mathbf{r}_{h_{1}} \mathbf{v}_{1}-\mathbf{r}_{h_{2}} \mathbf{v}_{1}\right)\right\|_{0, K}^{2} \lesssim h_{2}^{-1}\left\|\operatorname{curl}\left(\tilde{\mathbf{r}}_{h_{1}} \mathbf{v}_{1}-\tilde{\mathbf{r}}_{h_{2}} \mathbf{v}_{1}\right)\right\|_{0, \tau_{K}}^{2} .
$$


Using this estimate, we deduce from (4.23) that

$$
\begin{aligned}
\left\|\sum_{e \in \mathcal{E}_{h_{2}} \cap \Gamma_{12}} \lambda_{e}\left(\mathbf{r}_{h_{1}} \mathbf{v}_{1}-\mathbf{r}_{h_{2}} \mathbf{v}_{1}\right) \operatorname{curl} L_{e}\right\|_{0, \Omega_{2}}^{2} & \lesssim \sum_{K \subset \mathcal{T}_{21}}\left\|\operatorname{curl}\left(\tilde{\mathbf{r}}_{h_{1}} \mathbf{v}_{1}-\tilde{\mathbf{r}}_{h_{2}} \mathbf{v}_{1}\right)\right\|_{0, \tau_{K}}^{2} \\
& \lesssim\left\|\operatorname{curl}\left(\tilde{\mathbf{r}}_{h_{1}} \mathbf{v}_{1}-\tilde{\mathbf{r}}_{h_{2}} \mathbf{v}_{1}\right)\right\|_{0, \Omega_{1}}^{2} \\
& \lesssim h_{1}^{2 \delta_{1}}\left\|\operatorname{curl} \mathbf{c u r}_{1}\right\|_{\delta_{1}, \Omega_{1}}^{2}+h_{2}^{2 \delta_{1}}\left\|\operatorname{curl}_{1}\right\|_{\delta_{1}, \Omega_{1}}^{2} \\
& \lesssim h_{1}^{2 \delta_{1}}\left\|\operatorname{curl} \mathbf{v}_{1}\right\|_{\delta_{1}, \Omega_{1}} .
\end{aligned}
$$

This, along with (4.21) and Lemma 4.1 leads to

$$
\left\|\operatorname{curl}\left(\mathbf{r}_{h} \mathbf{v}-\mathbf{v}\right)\right\|_{0, \Omega_{2}}^{2} \lesssim h_{2}^{2 \delta_{2}}\left\|\operatorname{curl}_{2}\right\|_{\delta_{2}, \Omega_{1}}^{2}+h_{1}^{2 \delta_{1}}\left\|\operatorname{curlv}_{1}\right\|_{\delta_{1}, \Omega_{1}}^{2}
$$

Step 3. Estimate $\left\|\mathbf{r}_{h} \mathbf{v}-\mathbf{v}\right\|_{\operatorname{curl}, \Omega_{2}}$ when $\partial \Omega_{1} \cap \partial \Omega_{2}=\mathrm{E}_{12}$ is an edge. By the definition of $\mathbf{v}_{h_{2}}$, we know

$$
\mathbf{v}_{h_{2}}-\mathbf{v}_{2}=\left(\mathbf{r}_{h_{2}} \mathbf{v}_{2}-\mathbf{v}_{2}\right)+\mathbf{R}_{\mathrm{E}_{12}, h_{2}}^{0}\left(\left.\tilde{\mathbf{w}}_{h_{2}}\right|_{\mathrm{E}_{12}}\right)+\nabla p_{h_{2}} \text {. }
$$

We next estimate each term in (4.25). The first term can be estimated using Lemma 4.3

$$
\left\|\mathbf{r}_{h_{2}} \mathbf{v}_{2}-\mathbf{v}_{2}\right\|_{\text {curl }, \Omega_{2}}^{2} \lesssim h_{2}^{2 \delta_{2}}\left\|\operatorname{curl}_{2}\right\|_{\delta_{2}, \Omega_{2}}^{2} .
$$

To bound the second term in (4.25), as in Step 1 one can verify that $\left\|L_{e}\right\|_{0, \Omega_{2}}^{2} \lesssim h_{2}^{-1}$ and

$$
\begin{aligned}
\left\|\mathbf{R}_{\mathrm{E}_{12}, h_{2}}^{0}\left(\left.\tilde{\mathbf{w}}_{h_{2}}\right|_{\mathrm{E}_{12}}\right)\right\|_{0, \Omega_{2}}^{2} & \lesssim h_{2}^{2}\left\|\tilde{\mathbf{w}}_{h_{2}}\right\|_{0, \mathrm{E}_{12}}^{2}, \\
\left\|\operatorname{curl}_{\mathrm{R}_{12}, h_{2}}^{0}\left(\left.\tilde{\mathbf{w}}_{h_{2}}\right|_{\mathrm{E}_{12}}\right)\right\|_{0, \Omega_{2}}^{2} & \lesssim\left\|\tilde{\mathbf{w}}_{h_{2}}\right\|_{0, \mathrm{E}_{12}}^{2} .
\end{aligned}
$$

Then using Lemma 4.4. (4.6) and the fact that $\tilde{\mathbf{w}}_{h_{2}}=\tilde{\mathbf{r}}_{h_{2}} \mathbf{w}$, we derive

$$
\begin{aligned}
\left\|\mathbf{R}_{\mathrm{E}_{12}, h_{2}}^{0}\left(\tilde{\mathbf{w}}_{h_{2}} \mid \mathrm{E}_{12}\right)\right\|_{\text {curl }, \Omega_{2}}^{2} & \lesssim\left[1+\log \left(d / h_{2}\right)\right]\|\operatorname{curl} \mathbf{w}\|_{0, \Omega_{1}}^{2} \\
& =\left[1+\log \left(d / h_{2}\right)\right] \| \operatorname{curl}\left(\tilde{\mathbf{r}}_{h_{1}} \mathbf{v}_{1}-\tilde{\mathbf{r}}_{h_{2}} \mathbf{v}_{1} \|_{0, \Omega_{1}}^{2}\right. \\
& \lesssim h_{1}^{2 \delta_{1}}\left[1+\log \left(d / h_{2}\right)\right]\left\|\operatorname{curl} \mathbf{v}_{1}\right\|_{\delta_{1}, \Omega_{1}}^{2} .
\end{aligned}
$$

It remains to estimate the last term $\nabla p_{h_{2}}$ in (4.25). Noting that $p_{h_{2}}$ is discrete harmonic in $\Omega_{2}$, it follows from Lemma 4.6 that

$$
\begin{aligned}
\|\left.\nabla p_{h_{2}}\right|_{0, \Omega_{2}} ^{2} & \lesssim\left|p_{h_{2}}\right|_{\frac{1}{2}, \Gamma_{2}}^{2}=\left|\pi_{\mathrm{E}_{12}, h_{2}}^{0}\left(\left.\tilde{p}_{h_{2}}\right|_{\Gamma_{1}}\right)\right|_{\frac{1}{2}, \Gamma_{2}}^{2} \\
& \lesssim\left[1+\log \left(d / h_{1}\right)\right]\left|\tilde{p}_{h_{2}}\right|_{\frac{1}{2}, \Gamma_{1}}^{2} \lesssim\left[1+\log \left(d / h_{1}\right)\right]\left|\tilde{p}_{h_{2}}\right|_{1, \Omega_{1}}^{2},
\end{aligned}
$$

which, along with 4.11) and Lemma 4.3 yields

$$
\left\|\nabla p_{h_{2}}\right\|_{0, \Omega_{2}}^{2} \lesssim\left[1+\log \left(d / h_{2}\right)\right]\left\|\tilde{\mathbf{r}}_{h_{1}} \mathbf{v}_{1}-\tilde{\mathbf{r}}_{h_{2}} \mathbf{v}_{1}\right\|_{0, \Omega_{1}}^{2} \lesssim h_{1}^{2 \delta_{1}}\left[1+\log \left(d / h_{2}\right)\right]\left\|\operatorname{curl}_{1}\right\|_{\delta_{1}, \Omega_{1}}^{2} .
$$

Using (4.26)-(4.28), we derive the estimate for $\mathbf{v}_{h_{2}}-\mathbf{v}_{2}$ :

$$
\left\|\mathbf{v}_{h_{2}}-\mathbf{v}_{2}\right\|_{\text {curl }, \Omega_{2}}^{2} \lesssim h_{1}^{2 \delta_{1}}\left[1+\log \left(d / h_{2}\right)\right]\left\|\operatorname{curl}_{1}\right\|_{\delta_{1}, \Omega_{1}}^{2}+h_{2}^{2 \delta_{2}}\left\|\operatorname{curl}_{2}\right\|_{\delta_{2}, \Omega_{2}}^{2} .
$$

Now we can conclude (4.16) by combining (4.20), (4.24) and (4.29).

For the general $k>2$, we can show similarly as we did for $k=2$ above that

$$
\left\|\mathbf{r}_{h} \mathbf{v}-\mathbf{v}\right\|_{\mathbf{c u r l}, \Omega_{k}} \lesssim\left(\sum_{l \leq k, \partial \Omega_{l} \cap \partial \Omega_{k} \neq \emptyset} h_{l}^{2 \alpha_{l}}\|\mathbf{c u r l v}\|_{\delta_{l}, \Omega_{l}}^{2}\right)^{\frac{1}{2}} .
$$


Clearly, the error estimate (4.14) is now a direct consequence of (4.15), 4.16) and (4.30).

\section{NeARLy optimal ERROR ESTIMATES OF THE MORTAR ELEMENT METHOD}

This section is devoted to the establishment of a nearly optimal error estimate for the mortar edge element method proposed in Section 3. For this purpose, we first present three auxiliary results.

Lemma 5.1. For any $\varphi \in H^{\delta}\left(\operatorname{curl} ; \Omega_{k}\right)$ with $\delta>\frac{1}{2}$, the following Green's formula holds:

$\int_{\Omega_{k}} \operatorname{curl} \varphi \cdot \mathbf{v}_{h_{k}} d x-\int_{\Omega_{k}} \varphi \cdot \operatorname{curl} \mathbf{v}_{h_{k}} d x=\int_{\Gamma_{k}} \varphi \cdot\left(\mathbf{v}_{h_{k}} \times \mathbf{n}\right) d s, \quad \forall \mathbf{v}_{h_{k}} \in V_{h_{k}}\left(\Omega_{k}\right)$.

Proof. By the standard Helmholtz decomposition (4.1), we can find $\mathbf{w} \in\left(H^{1}\left(\Omega_{k}\right)\right)^{3}$ and $p \in H^{1}\left(\Omega_{k}\right)$ such that $\mathbf{v}_{h_{k}}=\mathbf{w}+\nabla p$. Then for any $\varphi \in H^{\delta}\left(\operatorname{curl} ; \Omega_{k}\right)$, we can write

$$
\begin{aligned}
& \int_{\Omega_{k}} \operatorname{curl} \varphi \cdot \mathbf{v}_{h_{k}} d x-\int_{\Omega_{k}} \varphi \cdot \operatorname{curl} \mathbf{v}_{h_{k}} d x \\
& \quad=\int_{\Omega_{k}} \operatorname{curl} \varphi \cdot \mathbf{w} d x-\int_{\Omega_{k}} \varphi \cdot \operatorname{curl} \mathbf{w} d x+\int_{\Omega_{k}} \operatorname{curl} \varphi \cdot \nabla p d x .
\end{aligned}
$$

Using the Sobolev embedding theorem, the fact that $\varphi \in H^{\delta}\left(\mathbf{c u r l} ; \Omega_{k}\right)$ implies $\left.\varphi\right|_{\Gamma_{k}} \in L^{2}\left(\Gamma_{k}\right)^{3}$ and $\left.(\operatorname{curl} \varphi \cdot \mathbf{n})\right|_{\Gamma_{k}} \in L^{2}\left(\Gamma_{k}\right)$. Thus the following relations hold:

$$
\begin{aligned}
& \int_{\Omega_{k}} \operatorname{curl} \varphi \cdot \nabla p d x=\int_{\Gamma_{k}}(\operatorname{curl} \varphi \cdot \mathbf{n}) p d s \\
& \int_{\Omega_{k}} \operatorname{curl} \varphi \cdot \mathbf{w} d x-\int_{\Omega_{k}} \varphi \cdot \operatorname{curl} \mathbf{w} d x=\int_{\Gamma_{k}} \varphi \cdot(\mathbf{w} \times \mathbf{n}) d s .
\end{aligned}
$$

Let $\left\{\Gamma_{k r}\right\}_{r=1}^{4}$ be the faces of $\Gamma_{k}$, and $\operatorname{curl}_{\Gamma_{k r}}$ and $\operatorname{curl}_{\Gamma_{k r}}$ the two-dimensional scalar and vector-valued curl-operators on $\Gamma_{k r}$ (cf. [17]). Then we have

$$
\int_{\Gamma_{k}}(\operatorname{curl} \varphi \cdot \mathbf{n}) p d s=\sum_{r=1}^{4} \int_{\Gamma_{k r}}(\operatorname{curl} \varphi \cdot \mathbf{n}) p d s=\sum_{\Gamma_{k r}} \int_{\Gamma_{k r}} \operatorname{curl}_{\Gamma_{k r}}\left(\left.\varphi\right|_{\Gamma_{k r}}\right) p d s .
$$

Note that $\nabla p=\mathbf{v}_{h_{k}}-\mathbf{w}$, so $\left.(\nabla p)\right|_{\Gamma_{k}} \in\left(L^{2}\left(\Gamma_{k}\right)\right)^{3}$. This proves $\left.p\right|_{\Gamma_{k}} \in H^{1}\left(\Gamma_{k}\right)$. Thus by Stokes' formula (cf. [17]), we have

$$
\int_{\Gamma_{k}}(\operatorname{curl} \varphi \cdot \mathbf{n}) p d s=\sum_{\Gamma_{k r}}\left[\int_{\Gamma_{k r}}\left(\operatorname{curl}_{\Gamma_{k r}} p\right) \cdot \varphi d s-\int_{\partial \Gamma_{k r}}(\varphi \cdot \mathbf{t}) p d s\right] .
$$

As $\varphi \in H^{\delta}\left(\operatorname{curl} ; \Omega_{k}\right)$ with $\delta>1 / 2$, we know that $\varphi$ has continuous tangential components across each edge of the polyhedron $\Omega_{k}$. So we derive from (5.5) that

$$
\int_{\Gamma_{k}}(\operatorname{curl} \varphi \cdot \mathbf{n}) p d s=\sum_{\Gamma_{k r}} \int_{\Gamma_{k r}}(\nabla p \times \mathbf{n}) \cdot \varphi d s=\int_{\Gamma_{k}} \varphi \cdot(\nabla p \times \mathbf{n}) d s .
$$

This, together with (5.3), leads to

$$
\int_{\Omega_{k}} \operatorname{curl} \varphi \cdot \nabla p d x=\int_{\Gamma_{k}} \varphi \cdot(\nabla p \times \mathbf{n}) d s .
$$

Now (5.1) follows from this, (5.4) and (5.2). 
Lemma 5.2. For any $\mathbf{v} \in V_{h}(\Omega)$, we have $\mathbf{v}_{i} \times \mathbf{n}=\mathbf{v}_{j} \times \mathbf{n}$ on $\Gamma_{i j}$ for each face $\Gamma_{i j}$ in Group 2.

Proof. Recall that there are two triangulations $\mathcal{T}_{h_{i}}$ and $\mathcal{T}_{h_{j}}$ on any face $\Gamma_{i j}$. If a face $\Gamma_{i j}$ is in Group 2, then $\mathcal{T}_{h_{i}}$ and $\mathcal{T}_{h_{j}}$ matches with each other. Since $\mathbf{v} \in V_{h}(\Omega)$, we have $\left.\left(\mathbf{v}_{i} \times \mathbf{n}-\mathbf{v}_{j} \times \mathbf{n}\right)\right|_{\Gamma_{i j}} \in V_{h}\left(\Gamma_{i j}\right)$ and $\mathbf{v}_{i} \times \mathbf{n}=\mathbf{v}_{j} \times \mathbf{n}$ on $\partial \Gamma_{i j}$. Thus $\left.\left(\mathbf{v}_{i} \times \mathbf{n}-\mathbf{v}_{j} \times \mathbf{n}\right)\right|_{\Gamma_{i j}} \in W\left(\Gamma_{i j}\right)$. Then the weak continuity conditions in the definition of $V_{h}(\Omega)$ implies

$$
\left\|\mathbf{v}_{i} \times \mathbf{n}-\mathbf{v}_{j} \times \mathbf{n}\right\|_{0, \Gamma_{i j}}^{2}=\left\langle\mathbf{v}_{i} \times \mathbf{n}-\mathbf{v}_{j} \times \mathbf{n}, \mathbf{v}_{i} \times \mathbf{n}-\mathbf{v}_{j} \times \mathbf{n}\right\rangle_{\Gamma_{i j}}=0,
$$

that shows $\mathbf{v}_{i} \times \mathbf{n}=\mathbf{v}_{j} \times \mathbf{n}$ on $\Gamma_{i j}$.

For $\mathbf{v} \in H\left(\operatorname{curl} ; \Omega_{k}\right)$, we use $\operatorname{div}_{\tau}\left(\mathbf{v} \times\left.\mathbf{n}_{k}\right|_{\Gamma_{k}}\right)$ to denote its tangential divergence. The next result about the tangential divergence can be found in [2].

Lemma 5.3. For any $\mathbf{v} \in H\left(\operatorname{curl} ; \Omega_{k}\right)$, the following estimate holds:

$$
\left\|\mathbf{v} \times \mathbf{n}_{k}\right\|_{-\frac{1}{2}, \Gamma_{k}}+\left\|\operatorname{div}_{\tau}\left(\mathbf{v} \times\left.\mathbf{n}_{k}\right|_{\Gamma_{k}}\right)\right\|_{-\frac{1}{2}, \Gamma_{k}} \lesssim\|\mathbf{v}\|_{\operatorname{curl}, \Omega_{k}}
$$

Now we are ready to establish our nearly optimal error estimate. We shall use the notation

$$
\|\mathbf{v}\|=\left(\sum_{k=1}^{N}\|\mathbf{v}\|_{\mathbf{c u r l}, \Omega_{k}}^{2}\right)^{\frac{1}{2}}, \quad \forall \mathbf{v} \in \prod_{k=1}^{N} H\left(\operatorname{curl} ; \Omega_{k}\right) .
$$

Theorem 5.1. Let $\mathbf{u}$ be the solution to the variational problem (2.3) and $\mathbf{u}_{h}$ be the finite element solution to the mortar element system (3.1). Assume that in the entire domain $\Omega$, we have the regularities $\mathbf{u} \in H(\mathbf{c u r l} ; \Omega)$ and $\mathbf{f} \in\left(H^{\delta}(\Omega)\right)^{3}$ with $\delta>\frac{1}{2}$, while in each subdomain we have $\left.\mathbf{u}\right|_{\Omega_{k}} \in H^{\delta_{k}}\left(\mathbf{c u r l} ; \Omega_{k}\right)$ and $\left.\mathbf{f}\right|_{\Omega_{k}} \in$ $\left(H^{\delta_{k}}\left(\Omega_{k}\right)\right)^{3}$ with $\delta_{k} \in\left(\frac{1}{2}, 1\right]$. Then

$$
\left\|\mathbf{u}_{h}-\mathbf{u}\right\| \lesssim[1+\log (d / h)]^{\frac{1}{2}}\left(\sum_{k=1}^{N} h_{k}^{2 \delta_{k}}\|\mathbf{c u r l} \mathbf{u}\|_{\delta_{k}, \Omega_{k}}^{2}\right)^{\frac{1}{2}} .
$$

Proof. As in the second Strang lemma, one can verify that

$$
\begin{aligned}
\left\|\mathbf{u}_{h}-\mathbf{u}\right\| & \leq \inf _{\mathbf{v}_{h} \in V_{h}(\Omega)}\left\|\mathbf{v}_{h}-\mathbf{u}\right\|+\sup _{\mathbf{w}_{h} \in V_{h}(\Omega)} \frac{\left|\sum_{k=1}^{N}\left[A_{k}\left(\mathbf{u}_{k}, \mathbf{w}_{h_{k}}\right)-\left(\mathbf{f}, \mathbf{w}_{h_{k}}\right) \Omega_{k}\right]\right|}{\left\|\mathbf{w}_{h}\right\|} \\
& \equiv(\mathrm{I})_{1}+(\mathrm{I})_{2} .
\end{aligned}
$$

Next we shall estimate $(\mathrm{I})_{1}$ and $(\mathrm{I})_{2}$.

Note that $(\mathrm{I})_{1}$ represents the approximation error and can be easily estimated using the generalized interpolation operator $\mathbf{r}_{h}$ defined in Section 4 . In fact, It follows from Lemma 4.2 and Theorem 4.1 that $\mathbf{r}_{h} \mathbf{u} \in V_{h}(\Omega)$ and

$$
\left\|\mathbf{r}_{h} \mathbf{u}-\mathbf{u}\right\| \lesssim[1+\log (d / h)]^{\frac{1}{2}}\left(\sum_{k=1}^{N} h_{k}^{2 \delta_{k}}\left(\|\mathbf{u}\|_{\mathbf{c u r l}, \delta_{k}, \Omega_{k}}^{2}\right)^{\frac{1}{2}},\right.
$$

which shows

$$
(\mathrm{I})_{1} \lesssim[1+\log (d / h)]^{\frac{1}{2}}\left(\sum_{k=1}^{N} h_{k}^{2 \delta_{k}}\left(\|\mathbf{u}\|_{\mathbf{c u r l}, \delta_{k}, \Omega_{k}}^{2}\right)^{\frac{1}{2}}\right.
$$


Now, we estimate the consistency error $(\mathrm{I})_{2}$. Noting (1.1) and the assumptions on $\mathbf{u}_{k}=\left.\mathbf{u}\right|_{\Omega_{k}}$ and $\mathbf{f}_{k}=\left.\mathbf{f}\right|_{\Omega_{k}}$, we know $\alpha$ curlu $\in H^{\delta_{k}}\left(\mathbf{c u r l} ; \Omega_{k}\right)$. Then using Lemma 5.1

$$
\sum_{k=1}^{N}\left[A_{k}\left(\mathbf{u}_{k}, \mathbf{w}_{h_{k}}\right)-\left(\mathbf{w}_{h_{k}}\right)_{\Omega_{k}}\right]=\sum_{k=1}^{N} \int_{\Gamma_{k}}(\alpha \mathbf{c u r l u}) \cdot\left(\mathbf{w}_{h_{k}} \times \mathbf{n}_{k}\right) d s .
$$

Again noting (1.1), we know $\alpha$ curlu $\in H(\mathbf{c u r l} ; \Omega)$, so $\alpha \mathbf{c u r l u} \times \mathbf{n}$ is continuous across $\Gamma_{i j}$, and it follows then from (5.10) that

$$
\sum_{k=1}^{N}\left[A_{k}\left(\mathbf{u}_{k}, \mathbf{w}_{h_{k}}\right)-\left(\mathbf{f}, \mathbf{w}_{h_{k}}\right)_{\Omega_{k}}\right]=\sum_{\Gamma_{i j} \subset \Gamma} \int_{\Gamma_{i j}}(\alpha \mathbf{c u r l u}) \cdot\left(\mathbf{w}_{h_{i}} \times \mathbf{n}-\mathbf{w}_{h_{j}} \times \mathbf{n}\right) d s .
$$

For ease of notation, set $\overline{\mathbf{u}}=\alpha \mathbf{c u r l u}$. From the assumption and (1.1), we know that $\overline{\mathbf{u}} \in H(\mathbf{c u r l} ; \Omega) \cap \prod_{k=1}^{N} H^{\delta_{k}}\left(\mathbf{c u r l} ; \Omega_{k}\right)$ with $\delta_{k}>\frac{1}{2}$. Thus, $r_{h} \overline{\mathbf{u}}$ is well defined and $\mathbf{r}_{h} \overline{\mathbf{u}} \in H(\operatorname{curl} ; \Omega)$ by Lemma 4.2. It can be seen, by the definition of $\mathbf{r}_{h}$, that $\left.\left(\mathbf{n} \times \mathbf{r}_{h} \overline{\mathbf{u}} \times \mathbf{n}\right)\right|_{\Gamma_{i j}} \in W\left(\Gamma_{i j}\right)$ for the faces $\Gamma_{i j}$ in Group 1. Then noting $\mathbf{w}_{h} \in V_{h}(\Omega)$, we have for each face $\Gamma_{i j}$ in Group 1 that

$\int_{\Gamma_{i j}}\left(\mathbf{r}_{h} \overline{\mathbf{u}}\right) \cdot\left(\mathbf{w}_{h_{i}} \times \mathbf{n}-\mathbf{w}_{h_{j}} \times \mathbf{n}\right) d s=\int_{\Gamma_{i j}}\left(\mathbf{n} \times \mathbf{r}_{h} \overline{\mathbf{u}} \times \mathbf{n}\right) \cdot\left(\mathbf{w}_{h_{i}} \times \mathbf{n}-\mathbf{w}_{h_{j}} \times \mathbf{n}\right) d s=0$.

On the other hand, we know $\mathbf{w}_{h_{i}} \times \mathbf{n}=\mathbf{w}_{h_{j}} \times \mathbf{n}$ on $\Gamma_{i j}$ for all the faces $\Gamma_{i j}$ in Group 2 by Lemma 5.2, so (5.12) holds. Using this we derive from (5.11) that

$$
\sum_{k=1}^{N}\left[A_{k}\left(\mathbf{u}_{k}, \mathbf{w}_{h_{k}}\right)-\left(\mathbf{f}, \mathbf{w}_{h_{k}}\right)_{\Omega_{k}}\right]=\sum_{\Gamma_{i j} \subset \Gamma} \int_{\Gamma_{i j}}\left(\overline{\mathbf{u}}-\mathbf{r}_{h} \overline{\mathbf{u}}\right) \cdot\left(\mathbf{w}_{h_{i}} \times \mathbf{n}-\mathbf{w}_{h_{j}} \times \mathbf{n}\right) d s .
$$

Since $\mathbf{r}_{h} \overline{\mathbf{u}} \times \mathbf{n}$ is also continuous across each $\Gamma_{i j}$, we can rewrite (5.13) as follows:

$$
\sum_{k=1}^{N}\left[A_{k}\left(\mathbf{u}_{k}, \mathbf{w}_{h_{k}}\right)-\left(\mathbf{f}, w_{h_{k}}\right)_{\Omega_{k}}\right]=\sum_{k=1}^{N} \int_{\Gamma_{k}}\left(\overline{\mathbf{u}}-\mathbf{r}_{h} \overline{\mathbf{u}}\right) \cdot\left(\mathbf{w}_{h_{k}} \times \mathbf{n}_{k}\right) d s .
$$

Using the Helmholtz decomposition for $\mathbf{w}_{h_{k}}, \mathbf{w}_{h_{k}}=\mathbf{w}+\nabla p$, we easily see

$$
\begin{aligned}
& \int_{\Gamma_{k}}\left(\overline{\mathbf{u}}-\mathbf{r}_{h} \overline{\mathbf{u}}\right) \cdot\left(\mathbf{w}_{h_{k}} \times \mathbf{n}_{k}\right) d s \\
= & \int_{\Gamma_{k}}\left(\overline{\mathbf{u}}-\mathbf{r}_{h} \overline{\mathbf{u}}\right) \cdot\left(\mathbf{w} \times \mathbf{n}_{k}\right) d s+\int_{\Gamma_{k}}\left(\overline{\mathbf{u}}-\mathbf{r}_{h} \overline{\mathbf{u}}\right) \cdot\left(\nabla p \times \mathbf{n}_{k}\right) d s \\
= & \int_{\Gamma_{k}}\left(\overline{\mathbf{u}}-\mathbf{r}_{h} \overline{\mathbf{u}}\right) \times \mathbf{n}_{k} \cdot \mathbf{w} d s+\int_{\Gamma_{k}} \operatorname{div}_{\tau}\left(\left(\overline{\mathbf{u}}-\mathbf{r}_{h} \overline{\mathbf{u}}\right) \times\left.\mathbf{n}_{k}\right|_{\Gamma_{k}}\right) p d s .
\end{aligned}
$$

Then we obtain by the Cauchy-Schwarz inequality and Lemma 5.3 that

$$
\begin{aligned}
\left|\int_{\Gamma_{k}}\left(\overline{\mathbf{u}}-\mathbf{r}_{h} \overline{\mathbf{u}}\right) \cdot\left(\mathbf{w}_{h_{k}} \times \mathbf{n}_{k}\right) d s\right| & \lesssim\left\|\left(\overline{\mathbf{u}}-\mathbf{r}_{h} \overline{\mathbf{u}}\right) \times \mathbf{n}_{k}\right\|_{-\frac{1}{2}, \Gamma_{k}} \cdot\|\mathbf{w}\|_{\frac{1}{2}, \Gamma_{k}} \\
& +\left\|\operatorname{div}_{\tau}\left(\left(\overline{\mathbf{u}}-\mathbf{r}_{h} \overline{\mathbf{u}}\right) \times\left.\mathbf{n}_{k}\right|_{\Gamma_{k}}\right)\right\|_{-\frac{1}{2}, \Gamma_{k}} \cdot\|p\|_{\frac{1}{2}, \Gamma_{k}} \\
& \lesssim\left\|\overline{\mathbf{u}}-\mathbf{r}_{h} \overline{\mathbf{u}}\right\|_{\operatorname{curl}, \Omega_{k}} \cdot\left(\|\mathbf{w}\|_{1, \Omega_{k}}+|p|_{1, \Omega_{k}}\right) .
\end{aligned}
$$

Using this and Lemma 4.5 we derive from (5.14) that

$$
\left|\sum_{k=1}^{N}\left[A_{k}\left(\mathbf{u}_{k}, \mathbf{w}_{h_{k}}\right)-\left(\mathbf{f}, \mathbf{w}_{h_{k}}\right)_{\Omega_{k}}\right]\right| \lesssim \sum_{k=1}^{N}\left\|\overline{\mathbf{u}}-\mathbf{r}_{a} \overline{\mathbf{u}}\right\|_{\operatorname{curl}, \Omega_{k}}\left\|\mathbf{w}_{h_{k}}\right\|_{\mathbf{c u r l}, \Omega_{k}} .
$$


Now by the Cauchy-Schwarz inequality and Theorem 4.1, we deduce

$$
\left|\sum_{k=1}^{N}\left[A_{k}\left(\mathbf{u}_{k}, \mathbf{w}_{h_{k}}\right)-\left(\mathbf{f}, \mathbf{w}_{h_{k}}\right) \Omega_{\Omega_{k}}\right]\right| \lesssim[1+\log (d / h)]^{\frac{1}{2}}\left(\sum_{k=1}^{N} h_{k}^{2 \delta_{k}}\|\mathbf{c u r l} \overline{\mathbf{u}}\|_{\delta_{k}, \Omega_{k}}^{2}\right)^{\frac{1}{2}} \cdot\left\|\mathbf{w}_{h}\right\| .
$$

This gives an estimate of $(\mathrm{I})_{2}$, which, along with (5.9) and (1.1), leads to the desired result.

Remark 5.1. Theorem 5.1 indicates a nearly optimal convergence of order $h^{\delta_{k}}(\log (d / h))^{1 / 2}$, for the newly proposed mortar edge element method under the natural regularity assumption on the exact solution $\mathbf{u} \in H(\mathbf{c u r l} ; \Omega)$ and $\left.\mathbf{u}\right|_{\Omega_{k}} \in$ $H^{\delta_{k}}\left(\mathbf{c u r l} ; \Omega_{k}\right)$ in each subdomain $\Omega_{k}$, with $\delta_{k}>1 / 2$. This is much improved compared to the requirement of the least regularity $\left.\mathbf{u}\right|_{\Omega_{k}} \in H^{2}\left(\mathbf{c u r l} ; \Omega_{k}\right)$ in order to achieve a nearly first order accuracy $h(\log (h))^{1 / 2}$ in [7] when the second family of Nedelec edge elements were used, and no convergence is possible under the regularity $\left.\mathbf{u}\right|_{\Omega_{k}} \in H^{1}\left(\mathbf{c u r l} ; \Omega_{k}\right)$. If the first family of Nedelec edge elements were used like we did in the current work, the convergence of the mortar edge element method of 7] will lose a further half order compared to the aforementioned convergence order of the second family. Also, unlike in [7, the ratio between the largest mesh size of all subdomains and the smallest one of all subdomains does not go into the bound of our final error estimate.

\section{NumERICAL EXPERIMENTS}

In this section we shall conduct some numerical experiments to check the convergence of the newly proposed mortar edge element method, and find out whether they are consistent with the prediction of the convergence theory developed in the previous sections.

For the convenience of computing the exact errors, we construct an example which has an exact solution. The coefficients and the domain in the system (1.1) will be taken as

$$
\alpha(x, y, z)=\beta(x, y, z)=1, \quad \Omega=(0,1) \times(0,1) \times(0,1),
$$

while the exact solution $\mathbf{u}=\left(u_{1}, u_{2}, u_{3}\right)^{T}$ is taken to be

$$
\begin{aligned}
& u_{1}=x y z(x-1)(y-1)(z-1), \\
& u_{2}=\sin (\pi x) \sin (\pi y) \sin (\pi z), \\
& u_{3}=\left(1-e^{x}\right)\left(1-e^{x-1}\right)\left(1-e^{y}\right)\left(1-e^{y-1}\right)\left(1-e^{z}\right)\left(1-e^{z-1}\right),
\end{aligned}
$$

and the right-hand side $\mathbf{f}$ is computed using the above given data through equation (1.1).

Then we need to triangulate the domain $\Omega$ into subdomains $\left\{\Omega_{k}\right\}$. For this, we first partition the three edges of $\Omega$ on $x$-, $y$ - and $z$-axes into equally distributed $m$ subintervals, using which one can naturally generate $m^{3}$ equal smaller cubes of size $H=1 / m$. This yields the desired subdomain decomposition in our experiments.

Next, we further triangulate each subdomain $\Omega_{k}$ to get two different fine triangulations of size $h$ over the domain $\Omega, \mathcal{T}_{h}^{1}$ and $\mathcal{T}_{h}^{2}$, where $\mathcal{T}_{h}^{1}$ consists of matching grids while $\mathcal{T}_{h}^{2}$ consists of nonmatching grids. To generate $\mathcal{T}_{h}^{1}$, we divide each subdomain into $n^{3}$ equal smaller cubes of size $h=1 /(m n)$, in the same manner as done in the previous subdomain generation. Then $\mathcal{T}_{h}^{1}$ is obtained by triangulating each 
cube into 6 tetrahedra. For easy identification, we may denote the triangulation $\mathcal{T}_{h}^{1}$ as $m^{3}\left(n^{3}\right)$ below.

To generate the nonmatching grid $\mathcal{T}_{h}^{2}$, we do exactly the same as for $\mathcal{T}_{h}^{1}$ for all the subdomains, except for the last subdomain sitting at the vertex $(1,1,1)$. For the last subdomain, we divide it into $(2 n)^{3}$ equal smaller cubes of size $h=1 /(2 m n)$, then triangulate each small cube into 6 tetrahedra.

We will measure the convergence accuracy of the proposed mortar edge element method using the following relative energy-norm:

$$
r:=\frac{\left\|e_{h}\right\|_{\text {curl }}}{\left\|e_{2 h}\right\|_{\text {curl }}}, \quad\left\|e_{h}\right\|_{\text {curl }}^{2}:=\sum_{k}\left\|\mathbf{u}_{h}-\mathbf{u}\right\|_{\text {curl } ; \Omega_{k}}^{2}
$$

where $\mathbf{u}$ is the exact solution to the system (1.1) and $\mathbf{u}_{h}$ is the approximation of $\mathbf{u}$ generated by the mortar edge element method.

The following two tables present the convergence of the mortar edge element method with the matching and nonmatching grids $\mathcal{T}_{h}^{1}$ and $\mathcal{T}_{h}^{2}$ when the number of subdomains and the fine mesh size in each subdomain vary. From the data shown in the first three columns of Table 6.1 , we can clearly see the optimal first order convergence when the matching grid $\mathcal{T}_{h}^{1}$ is used. The data given in the 4 th to 6 th columns of Table 6.1 (also the data in the 7 th to 9 th columns) indicates that the optimal first order convergence is affected by the change of the ratio $d / h$ between the subdomain size $d$ and the fine mesh size $h=d / n$, as predicted by the convergence theory stated in Theorem 5.1 .

When the subdomain size $d$ reduces while the parameter $n=d / h$ is fixed, the factor $\log (d / h)=\log n$ appearing in the bound of the error estimate of Theorem 5.1 is also fixed, hence the convergence will not deteriorate as predicted by the convergence theory in Theorem 5.1. Table 6.2 clearly shows the optimal first order convergence in this case when the nonmatching grid $\mathcal{T}_{h}^{2}$ is used.

We remark that the scales of the mortar edge element systems tested in our experiments are very large, for example, the degrees of freedom for two cases with $m^{3}\left(n^{3}\right)=12^{3}\left(3^{3}\right)$ and $m^{3}\left(n^{3}\right)=3^{3}\left(12^{3}\right)$ in Tables 6.1 and 6.2 are 416142 and 421704 , respectively.

TABLE 6.1. Convergence with $n=2,4,8$ or $n=3,6,12$ but $m=3$ fixed.

\begin{tabular}{|c|c|c||c|c|c||c|c|c|}
\hline $\mathcal{T}_{h}^{1}$ & $\left\|e_{h}\right\|_{\text {curl }}$ & $r$ & $\mathcal{T}_{h}^{2}$ & $\left\|e_{h}\right\|_{\text {curl }}$ & $r$ & $\mathcal{T}_{h}^{2}$ & $\left\|e_{h}\right\|_{\text {curl }}$ & $r$ \\
\hline $3^{3}\left(2^{3}\right)$ & $3.828 \mathrm{e}-1$ & & $3^{3}\left(2^{3}\right)$ & $3.788 \mathrm{e}-1$ & & $3^{3}\left(3^{3}\right)$ & $2.560 \mathrm{e}-1$ & \\
\hline $3^{3}\left(4^{3}\right)$ & $1.934 \mathrm{e}-1$ & 0.505 & $3^{3}\left(4^{3}\right)$ & $1.937 \mathrm{e}-1$ & 0.511 & $3^{3}\left(6^{3}\right)$ & $1.315 \mathrm{e}-1$ & 0.514 \\
\hline $3^{3}\left(8^{3}\right)$ & $9.692 \mathrm{e}-2$ & 0.501 & $3^{3}\left(8^{3}\right)$ & $1.010 \mathrm{e}-1$ & 0.521 & $3^{3}\left(12^{3}\right)$ & $7.031 \mathrm{e}-2$ & 0.535 \\
\hline
\end{tabular}

TABle 6.2. Convergence with $m=3,6,12$ but $n=3$ fixed.

\begin{tabular}{|c|c|c|}
\hline $\mathcal{T}_{h}^{2}$ & $\left\|e_{h}\right\|_{\text {curl }}$ & $r$ \\
\hline $3^{3}\left(3^{3}\right)$ & $2.560 \mathrm{e}-1$ & \\
\hline $6^{3}\left(3^{3}\right)$ & $1.291 \mathrm{e}-1$ & 0.504 \\
\hline $12^{3}\left(3^{3}\right)$ & $6.463 \mathrm{e}-2$ & 0.501 \\
\hline
\end{tabular}




\section{ACKNOWLEDGMENTS}

The authors wish to thank one anonymous referee for many constructive comments which led to a great improvement of the paper.

\section{REFERENCES}

[1] A. Ben Abdallah, F. Ben Belgacem and Y. Maday, Mortaring the two-dimensional "Nedelec" finite elements for the discretization of the Maxwell equations. To appear in M3AS.

[2] A. Alonso and A. Valli, Some remarks on the characterization of the space of tangential traces of $H(\mathbf{c u r l} ; \Omega)$ and the construction of an extension operator, Manuscr. Math., 89(1996), 159178. MR 1371994 (96k:46057)

[3] A. Alonso and A. Valli, An optimal domain decomposition preconditioner for low-frequency time-harmonic Maxwell equations, Math. Comp., 68(1999), 607-631. MR1609607 (99i:78002)

[4] C. Amrouche, C. Bernardi, M. Dauge and V. Girault. Vector Potentials in three-dimensional nonsmooth domains, Math. Meth. Appl. Sci., 21(1998), 823-864. MR1626990 (99e:35037)

[5] D. Arnold, R. Falk and R. Winther. Multigrid in $H$ (div) and $H($ curl), Numer. Math., 85(2000), 175-195. MR1754719 (2001d:65161)

[6] F. Assous, P. Degond, E. Heintzé, P. Raviart and J. Segre, On a finite-element method for solving the three-dimensional Maxwell equations, J. Comput. Phys., 109(1993), 222-237. MR.1253460 (94j:78003)

[7] F. Ben Belgacem, A. Buffa and Y. Maday, The mortar finite element method for 3D Maxwell equations: first results, SIAM J. Numer. Anal., 39(2001), 880-901 MR.1860449 (2003i:78019)

[8] F. Ben Belgacem and Y. Maday, The mortar element method for three dimensional finite elements, $\mathrm{M}^{2} \mathrm{AN}$ 31(1997), 289-302. MR1437123 (98e:65107)

[9] C. Bernardi, Y. Maday and A. Patera, A new nonconforming approach to domain decomposition: the mortar element method. In: Nonlinear partial differential equations and their applications (H. Brezis and J. Lions (eds.)), Pitman, 1994.

[10] M. Birman and M. Solomyak, $L_{2}$-theory of the Maxwell operator in arbitrary domains, Russian Math. Surveys, 42(1987), 75-96. MR933995 (89e:35127)

[11] S. Börm and R. Hiptmair, Multigrid computation of axismmetric electromagnetic fields, Adv. Comp. Math., 16(2001), 331-356. MR.1894928 (2003d:78042)

[12] M. Cessenat, Mathematical methods in electromagnetism, World Scientific, River Edge, NJ, 1998 MR 1409140 (97j:78001)

[13] Z. Chen, Q. Du and J. Zou, Finite element methods with matching and nonmatching meshes for Maxwell equations with discontinuous coefficients, SIAM J. Numer. Anal., 37(2000), 1542-1570. MR1759906 (2001h:78044)

[14] P. Ciarlet, Jr. and J. Zou, Finite element convergence for the Darwin model to Maxwell's equations, RAIRO Math. Modelling Numer. Anal., 31(1997), 213-249. MR.1437121 (98b:78001)

[15] P. Ciarlet, Jr. and J. Zou, Fully discrete finite element approaches for time-dependent Maxwell's equations, Numer. Math., 82(1999), 193-219. MR1685459 (2000c:65083)

[16] R. Dautray and J.-L. Lions, Mathematical analysis and numerical methods for science and technology, Springer-Verlag, New York, 1988. MR0969367 (89m:00001)

[17] V. Girault and P. Raviart, Finite Element Methods for Navier-Stokes Equations, SpringerVerlag, Berlin, 1986. MR0851383 (88b:65129)

[18] J. Gopalakrishnan and J. Pasciak, Overlapping Schwarz preconditioners for indefinite time harmonic Maxwell's equations, Math. Comput., 72(2003), 1-16. MR.1933811(2003i:78020)

[19] R. Hiptmair, Multigrid method for Maxwell's equations, SIAM J. Numer. Anal., 36(1998), 204-225. MR1654571 (99j:65229)

[20] R. Hiptmair, Finite elements in computational electromagnetism, Acta Numerica, 11(2002), 237-339. MR2009375 (2004k:78028)

[21] R. Hoppe, Mortar edge element methods in $R^{3}$, East-West J. Numer. Anal., 7(1999), 159-173. MR.1719615 (2001c:65155)

[22] Q. Hu, Numerical Integrations and Unit Resolution Multipliers for Domain Decomposition Methods with Nonmatching Grids, Computing, 74(2005), 101-129. MR2133691(2005k:65275) 
[23] Q. Hu, G. Liang and J. Lui, The construction of preconditioner for domain decomposition methods with polynomial multipliers, J. Comp. Math., 19(2001), 213-224 MR1816686 (2001k:65186)

[24] Q. Hu and J. Zou, Two new variants of nonlinear inexact Uzawa algorithms for saddle-point problems, Numer. Math., 93 (2002), 333-359. MR1941400(2004c:65029)

[25] Q. Hu and J. Zou, Nonlinear inexact Uzawa algorithms for linear and nonlinear saddle-point problems, SIAM J. Optim., 16 (2006), 798-825. MR2197557 (2006j:65087)

[26] Q. Hu and J. Zou, A nonoverlapping domain decomposition method for Maxwell's equations in three dimensions, SIAM J. Numer. Anal., 41(2003), 1682-1708. MR2035002 (2005a:65131)

[27] Q. Hu and J. Zou, Substructuring preconditioners for saddle-point problems arising from Maxwell's equations in three dimensions, Math. Comput., 73(2004), 35-61. MR2034110 (2004m:65197)

[28] C. Kim, R. Lazarov, J. Pasciak and P. Vassilevski, Multiplier spaces for the mortar finite element method in three dimensions, SIAM J. Numer. Anal., 39 (2001), 517-538. MR1860265 (2002g:65143)

[29] G. Liang and J. He, The non-conforming domain decomposition method for elliptic problems with Lagrangian multipliers, Chinese J. Num. Math. Appl.15:1(1993), 8-19. MR.1600626

[30] P. Monk, Analysis of a finite element method for Maxwell's equations, SIAM J. Numer. Anal., 29(1992), 32-56. MR.1163353 (93k:65096)

[31] J. Nédélec, Mixed finite elements in $R^{3}$, Numer. Math., 35(1980), 315-341. MR592160 (81k:65125)

[32] R. Nicolaides and D. Wang, Convergence analysis of a covolume scheme for Maxwell's equations in three dimensions, Math. Comp., 67(1998), 947-963. MR.1474654 (98j:65080)

[33] J. Pasciak and J. Zhao, Overlapping Schwarz methods in H (curl) on nonconvex domains, East-West J. Numer. Anal., 10(2002), 221-234. MR1935967(2003j:65108)

[34] A. Toselli and A. Klawonn, A FETI domain decomposition method for edge element approximations in two dimensions with discontinuous coefficients, SIAM J. Numer. Anal., 39(2001), 932-956. MR1860451 (2002f:65169)

[35] A. Toselli, O. Widlund and B. Wohlmuth, A FETI preconditioner for two dimensional edge element approximations of Maxwell's equations on nonmatching grids, SIAM J. Sci. Comput., 23(2001), 92-108. MR1860906 (2002m:65025)

[36] B. Wohlmuth, A mortar finite element method using dual spaces for the Lagrange multiplier, SIAM J. Numer. Anal., 38(2000), 989-1012. MR1781212 (2001h:65132)

[37] J. Xu and J. Zou, Some nonoverlapping domain decomposition methods, SIAM Review, 40(1998), 857-914. MR1659681 (99m:65241)

LSEC and Institute of Computational Mathematics and Scientific/Engineering Computing, Academy of Mathematical and System Sciences, The Chinese Academy of SciENCES, BeiJing 100080, China

E-mail address: hqy@lsec.cc.ac.cn

School of Mathematics and Computational Science, Xiangtan University, Xiangtan, Hunan 411105, China

E-mail address: shushi@xtu.edu.cn

Department of Mathematics, The Chinese University of Hong Kong, Shatin, N.T., HONG KONG

E-mail address: zou@math.cuhk.edu.hk 